\title{
Host Galaxy Evolution in Radio-Loud AGN
}

\author{
Matthew O'Dowd \\ School of Physics, University of Melbourne, Parkville, Victoria 3010, Australia; \\ modowd@physics.unimelb.edu.au \\ C. Megan Urry \\ Department of Physics and Yale Center for Astronomy and Astrophysics, P.O. Box \\ 208121, New Haven, CT 06520-8121, USA; meg.urry@yale.edu
}

\begin{abstract}
We investigate the luminosity evolution of the host galaxies of radio-loud AGN through Hubble Space Telescope imaging of 72 BL Lac objects, including new STIS imaging of nine $z>0.6$ BL Lacs. With their intrinsically low accretion rates and their strongly beamed jets, BL Lacs provide a unique opportunity to probe host galaxy evolution independent of the biases and ambiguities implicit in quasar studies. We find that the host galaxies of BL Lacs evolve strongly, consistent with passive evolution from a period of active star formation in the range $0.5 \lesssim z \lesssim$ 2.5, and inconsistent with either passive evolution from a high formation redshift or a non-evolving population. This evolution is broadly consistent with that observed in the hosts of other radio-loud AGN, and inconsistent with the flatter luminosity evolution of quiescent early types and radio-quiet hosts. This indicates that active star formation, and hence galaxy interactions, are associated with the formation for radio-loud AGN, and that these host galaxies preferentially accrete less material after their formation epoch than galaxies without powerful radio jets. We discuss possible explanations for the link between merger history and the incidence of a radio jet.
\end{abstract}

Subject headings: galaxies: active - BL Lacertae objects: general - quasars: general — galaxies: evolution — galaxies: jets — black hole physics

\section{Introduction}

From Hubble Space Telescope (HST) studies of local galaxies, it appears that nearly all contain supermassive black holes (SMBHs) (Joseph et al. 2001; Bower et al. 2001; Sarzi 
et al. 2001; Barth et al. 2001; Gebhardt et al. 2003). Combined with the predictions from hierarchical galaxy formation, this suggests that the formation and evolution of galaxies and SMBHs might be closely linked (Silk \& Rees 1998; Haehnelt \& Rees 1993; Kauffmann \& Haehnelt 2000; Franceschini et al. 1999; Merrifield et al. 2000; Wang, Biermann \& Wandel 2000; di Matteo et al 2003). Circumstantial evidence also points to a direct link between the evolution of normal galaxies with the AGN phenomenon - the co-moving densities of both rise rapidly from today back to redshift $\sim 2$ (Schmidt \& Green 1983; Boyle, Shanks \& Peterson 1988; Shaver et al. 1996; Lilly et al. 1995; Driver et al. 1995; Cowie et al. 1996; Madau et al. 1996; Connolly et al. 1997; Cowie et al 2003; Hasinger 2003). Certainly it is plausible that AGN luminosity affects host galaxy properties, particularly star formation rates (e.g. de Young 1989; Rees 1989; Whittle 1992; Gonzalez Delgado 1995; Barthel 2001; Zirm, Dickinson \& Dey 2003), and a number of studies have found a dependence of maximum AGN power on host galaxy luminosity (Smith et al. 1986; Veŕon-Cetty \& Woltjer 1990; Hutchings et al. 1984; Hooper, Impey \& Foltz 1997; McLeod, Rieke \& Storrie-Lombardi 1999; Schade, Boyle \& Letawsky 2000; Dunlop et al. 2003).

It may be that most galaxies pass through one or more active phases at some point in their development (Cavaliere \& Padovani 1989). If so, then comparing the luminosity evolution of AGN host galaxies to that of normal galaxies allows us to study the link between AGN activity and galaxy evolution. If galaxy interactions are an important trigger of AGN activity (e.g. Hutchings \& Campbell 1983; Heckman et al. 1984; Yee 1987; Heisler 1991; Hutchings \& Neff 1992; Canalizo \& Stockton 2001) then there is a link between AGN activity and the assembly of elliptical galaxies, which should be reflected in the luminosity evolution of AGN host galaxies (Franceschini et al. 1999).

Work on host galaxy evolution to date has largely been restricted to high-power sources due to the difficulty in finding low-power AGN at sufficiently high redshift for the evolution of galaxies to become significant. The host galaxies of powerful radio-loud AGN at high redshift appear to be brighter than general radio-loud host galaxies observed at low redshift, consistent with passively evolving stellar populations (Aragon-Salamanca et al. 1993; Lilly \& Longair 1982; Kukula et al. 2001; McLure \& Dunlop 2000; Lehnert et al. 1999). The same is not observed of brightest cluster galaxies (BCGs), which exhibit flat luminosity evolution over the redshift range 0 to 1 (Aragon-Salamanca et al. 1993), and so are likely to be gaining in luminous mass. This is also tentatively the case for other early-type galaxies (Stanford et al. 1998; Lilly et al. 1995; Kauffmann et al. 1996; Bell et al. 2004). The host galaxies of radio-quiet AGN seem to exhibit similar flat or negative luminosity evolution, and appear to be gaining mass between redshift $z \sim 2$ and the present (Kukula et al. 2001; Rix et al. 2001; Ridgway et al. 2001). 
However, various biases make it impossible to confidently ascribe the observed evolution to the host galaxies themselves. First, it appears that maximum AGN power increases with host mass (Smith et al. 1986; Veŕon-Cetty \& Woltjer 1990; Hutchings et al. 1984; Hooper, Impey \& Foltz 1997; Schade, Boyle \& Letawsky 2000; McLeod, Rieke \& Storrie-Lombardi 1999; Scarpa \& Urry 2001; O’Dowd, Urry \& Scarpa 2002; Dunlop et al. 2003). This may induce apparent redshift evolution in flux-limited samples. Second, in powerful AGN, scattered light from the nucleus and extended, ionized gas contaminates host galaxy light. In radio galaxies, nuclear emission may be mistaken for host galaxy light (Dunlop \& Peacock 1993). This contamination increases with AGN power, which may again produce a spurious host luminosity-redshift trend.

BL Lac objects are intrinsically low-power AGN, with much lower accretion rates than quasars (O'Dowd, Urry \& Scarpa 2002) or powerful radio galaxies, yet the relativistic beaming of their jet emission means that they can be detected in significant numbers to $z \sim 1$, at which point galaxy luminosity evolution becomes measurable. Fortuitously, BL Lac objects overcome many of the potential biases outlined above.

First, the apparent luminosity of BL Lac objects is dominated by magnified emission from their relativistically beamed aligned jets (Urry \& Padovani 1995). This magnification is in turn dominated by the angle of the jet to the line of sight. Variation in jet angle washes out any trend between the power of the nucleus and the luminosity of the host galaxy (supported by absence of any correlation between host luminosity and beamed nuclear luminosity; Urry et al. 2000.) Second, the host galaxies of BL Lac objects are less likely to suffer from contamination by scattered light from their low-power nuclei, nor do they tend to exhibit the luminous, extended, ionized gas observed in more powerful AGN.

Due to the $(1+z)^{4}$ surface brightness dimming of the hosts (compared to $(1+z)^{3}$ dimming of the nucleus), the Hubble Space Telescope (HST) is needed to reliably determine BL Lac host galaxy parameters beyond $z \sim 0.6$. In this paper, we present new HST ${ }^{1}$ STIS images of high-redshift BL Lac objects. Combined with earlier $z<0.6$ HST WFPC2 observations, we measure the evolution of radio-loud AGN host galaxies, free from many of the biases implicit in previous studies. The observations and data analysis are described in $\S 2$. Results on the host galaxy properties are in $\S 3$, analysis and results for the host galaxy luminosity evolution are presented in $\S 4$, and the discussion of these results in $\S 5$. Conclusions are summarised in $\S 6$.

\footnotetext{
${ }^{1}$ Based on observations with the NASA/ESA Hubble Space Telescope, obtained at the Space Telescope Science Institute, which is operated by the Association of Universities for Research in Astronomy, Inc. under NASA contract No. NAS5-26555.
} 


\section{Observations and Data Analysis}

\subsection{The Sample}

The new STIS observations are high-redshift BL Lac objects selected from the cycle 6 HST snapshot survey of Urry et al. (2000). The original 132 proposed snapshot targets were chosen from six flux-limited samples. These were essentially all known BL Lac objects in complete samples in 1995. The 110 BL Lacs finally imaged in the snapshot program were chosen at random from the larger sample, and are an unbiased subset. The sample chosen

for STIS imaging comprised the 9 least optically luminous BL Lacs with $z>0.6$, spanning a range at least to $z \sim 1$. As observed nuclear brightness is dominated by variation in jet angle, this luminosity requirement does not introduce significant bias, but does dramatically increase our chances of detecting the host galaxy. Table 1 gives the final target list and exposure information, with the magnitudes and host galaxy limits derived from the WFPC2 snapshot images.

These targets were imaged with the STIS camera aboard HST, using the F28 $\times 50 \mathrm{LP}$ longpass filter. This configuration maximizes sensitivity to the host galaxy, sampling from $5,500 \AA$ to $10,000 \AA$. The absence in BL Lac objects of a big blue bump or extended line emission, as seen in quasars, makes it possible to use such a broad filter.

\subsection{Data Reduction and Calibration}

Four to five exposures were taken for each target, dithered in a square with edge length 5 pixels. In each case, one exposure was short enough to ensure that the nucleus was not saturated. The data were reduced using the STIS package in IRAF's STSDAS suite. The bias and dark frames were subtracted and the images flat-fielded using the routine STIS reduction process. CRREJ was used to perform cosmic ray rejection.

Most of the long exposures resulted in over-exposure and bleeding of the BL Lac nuclei. In each of these cases the affected pixels in the combined image were flagged and replaced with the pixels in the shorter exposure, normalised according to their exposure times.

\subsection{Sky Subtraction}

Accurate determination of the background level and its uncertainty is critical in this analysis, as an incorrect measurement can easily lead to the false detection of host galaxies. 
The background was taken to be the median in the region of each combined imaged which

was unaffected by any visible source. Such sources were masked well beyond their visible extent for determination of the background.

The error in the background value was calculated from two contributions: the pixelto-pixel Poissonian shot noise $\left(\sigma_{\text {Poisson }}\right)$, and the large-scale variation in the background level $\left(\sigma_{\text {large-scale }}\right)$. The latter was taken to be the maximum of: standard deviation in the medians of a grid of $20 \times 20$ pixel squares, and the standard deviation in the medians of the four quadrants of the image. The final error in the subtracted sky value was then:

$$
\sigma_{\text {sky }}=\sqrt{\sigma_{\text {Poisson }}^{2}+\sigma_{\text {large-scale }}^{2}}
$$

Figure 1 shows the central $300 \times 300$ pixels of the combined, sky-subtracted images. Also shown are the contour plots of the regions surrounding the targets, with north indicated by the arrow head and east indicated by the arrow tail.

\subsection{Modelling the STIS Point Spread Function}

Perhaps the most critical step in extracting the properties of the host galaxies is the accurate modelling of the Point Spread Function (PSF). Unlike ground-based PSFs, the HST PSF is relatively stable with time, and so synchronous PSF observations are not essential. However, the PSF does vary significantly across the surface of the chip, and this must be taken into account. In all images the target was within 20 pixels of the centre, so such spatial variations are minimized.

Due to deficiencies in the Tiny Tim model of the STIS PSF (it fails to account for extended, scattered light, the STIS 'ghost loop' and an asymmetric bulge near the PSF's centre), we elected to model the PSF with archival images of well-centred stars taken with STIS in the F $28 \times 50 \mathrm{LP}$ filter. Highly over-exposed stellar images were used to model the PSF wings, while unsaturated images were used to model the central regions. Figure 2 shows the final composite stellar PSF. The 'ghost loop', a reflection feature in the STIS PSF, was found to vary significantly even with small positional offsets, and so we simply mask this feature in all of the analysis to follow.

Given the potential inaccuracies in the STIS PSF extreme care was taken to account for its uncertainties. Deviations in the PSF arise from spatial variations, time variations, and spectral variations. The stars that were averaged to make our composite PSF have different spectral types, were taken at different times, and are offset from each other spatially 
(although all are still relatively close to the centre). Thus, the deviations observed among these PSFs should reflect the potential difference between the composite PSF and our data. We define the uncertainty in each pixel of the composite PSF model to be the maximum difference between that pixel value and the corresponding pixels in the component stellar images. This gave a conservative uncertainty map which was used in the two-dimensional host galaxy fitting.

\subsection{Host Galaxy Fitting}

Host galaxy properties were measured using two-dimensional model fitting. The models comprised the stellar PSF (§.2.4) centred on an analytic galaxy model. The host galaxies were modelled with the following profile types: a de Vaucouleurs $r^{1 / 4}$ law, an exponential disk $r^{-1}$ law, and a composite de Vaucouleurs plus exponential disk model. The free parameters fitted for each host galaxy profile type were: PSF normalization, host galaxy normalization (two normalizations for the composite model), host galaxy characteristic radius (again, two parameters for the composite model), ellipticity, and position angle.

Certain parameters were limited or fixed, as the quality of the data did not justify more detailed fitting. The point source was fixed at the centre of the galaxy, as this is observed to be the case all in low redshift BL Lacs (Urry et al. 2000). The galaxy models were limited to the three profile types described, as we are primarily interested in distinguishing between disk- and bulge-dominated morphologies. Modelling more detailed profiles (such as via the Sersic index) is beyond the scope of these data.

Before fitting, images were carefully masked to eliminate all irregular features such as close companions and tidal structure, along with several pixels beyond the visible edges of these features. The centre-most few pixels of the nucleus are highly sensitive to sampling errors. The values of these pixels will depend strongly on exactly where in the pixel the point-like nucleus falls. If the model PSF is offset even slightly from this (by as much as 0.05 of a pixel), large errors will occur in the fit, as these central pixels strongly weight our fitting statistic. To remedy this, we sum the pixel values within a central circle with a 7 -pixel diameter, and treat this as a single data point. As a side-benefit, this process greatly reduces the potential error due to an inaccurately modelled PSF.

In all cases but that of $2240-260$, the fits indicated the presence host galaxies. The residuals of $0820+225,1144-379,1249+174,1422+580$ and $1533+535$ were smooth and either circularly or elliptically symmetric about the nucleus. The residuals of 0138-097, $0235+164$ and $1308+326$ were less convincing, so great care was taken to determine the 
confidence of the detections.

\subsubsection{Detection Criteria and Upper Limits}

To determine whether the apparent host galaxy detections were significant, we fitted each image with the PSF alone, and compared the $\chi^{2}$ values of the best PSF-only fit to the best PSF + host galaxy fit via the F-test. We required that the model including the host galaxy be preferred over the PSF-only fit with $99 \%$ confidence.

To make certain that we did not mistake improperly subtracted background light for the host galaxy, we also perform the fits with the background over-subtracted by $2 \sigma_{\text {sky }}$ (see $\S 2.3$ ), and required that the model including the host galaxy was preferred over the PSF-only fit with $95 \%$ confidence in this case.

To ensure that none of the detected host galaxies were simply artifacts of a poorlymodelled PSF, we redid the fits using each of the individual stellar images as our PSF model instead of the combined stellar PSF, and required again that the host galaxy detected with each fit was preferred over the PSF-only fit with $99 \%$ confidence. For each alternate PSF, we again require the host galaxy be preferred at $95 \%$ with the background over-subtracted.

After applying these requirements, it was found that five of the nine host galaxy were detected with high confidence. These were the host galaxies of $0820+225,1144-379,1249+174$, $1422+580$ and $1533+535$. Of the remainder, 0138-097, 0235+164 and 1308+326 yielded host galaxies in the initial fit, but these did not meet our confidence requirements when the background was over-subtracted and/or the alternate PSFs used. 2240-260 did not show a detectable host galaxy in any of the fits.

Figure 3 shows the one-dimensional, azimuthally averaged profiles of both the data and the best fits. Galaxy profiles are presented only in cases where the PSF + host galaxy model is preferred over the PSF-only model according to the initial resolution criterion; ie. for 0138-097 and 0235+164 and the exponential disk fit of 1308+326, where the model was

rejected only after increasing the sky background or after fitting with an alternate PSF, we still present the best-fit PSF + galaxy profiles.

Figure 4 shows the images with PSF subtracted. For the detections we use the best PSF normalization from the de Vaucouleurs fit, and for the non-detections we use the best PSF-only fit. For the detections we also show the images with best-fit PSF + de Vaucouleurs model subtracted.

As a last check, we take the ratio of light in a aperture of radius 3.5 pixels around the 
centre to the light an annulus from 15 to 50 pixels, and compare to the same ratio for the PSF. In all five of the confident detections, the BL Lac image showed a significant excess of light in the annulus, indicating the presence of host galaxies. In the non-detections the excess was marginal.

For the non-detections, we determined the upper limit on the host galaxy magnitude by finding the maximum amount of host galaxy which could be added before the PSF + host galaxy fit became worse than the PSF-only fit at the 99\% confidence level by an F-test. In these cases, the galaxy model used was a de Vaucouleurs model with zero ellipticity and an effective radius of $10 \mathrm{kpc}$ - slightly higher than the median found for the low redshift BL Lac hosts of $8.5 \mathrm{kpc}$ (Urry et al. 2000). This is conservative, as it will give a slightly higher upper limit than for the median $r_{e}$.

\subsubsection{Uncertainty Analysis and Resolving Morphologies}

The uncertainty in each fitted parameter was determined by mapping $\chi^{2}$ around the best fit. To account for the uncertainty in the background subtraction, the fits were performed, and the $\chi^{2}$ volume was mapped with the background both under- and over-subtracted by $\sigma_{s k y}$ (see $\S 2.3$ ). The uncertainty in each parameter was then the maximum displacement from that parameter on the one-sigma hypersurface of the $\chi^{2}$ volume.

For a statistically significant preference of one profile type over the other, we again employ the F-test, requiring that their minimum $\chi^{2}$ values differ with $99 \%$ confidence. In most cases, the de Vaucouleurs model was marginally preferred over the exponential disk model, however in only two of these cases was the preference significant - for $0820+225$ and $1422+580$. In no cases was the exponential disk profile preferred over the de Vaucouleurs profile with high confidence.

As might be expected, the composite de Vaucouleurs + exponential disk model gave a better fit than either the de Vaucouleurs or exponential disk model alone. Typically, the improvement in the fit was marginal - at less than $90 \%$ confidence according to the F-test in all cases but $0820+225$, for which the three-component model was preferred over the best-fit two-component model with $\sim 90 \%$ confidence.

A number of the de Vaucouleurs model fits gave unphysically large effective radii, suggesting that the fit may be trying to compensate for an extended, disky component. Restricting the PSF + de Vaucouleurs model to more physical scale lengths - requiring $r_{e} \leq 20 \mathrm{kpc}$ - then the composite galaxy model is preferred over the new best-fit PSF + de Vaucouleurs model for $0820+225$ at the $99 \%$ confidence level. If we apply the same condition to the rest of 
the sample, both $1422+580$ and $1533+535$ prefer the composite model at $\sim 95 \%$ confidence, and $1144-379$ at the $\sim 90 \%$ level.

Figure 3 shows $\chi^{2}$ contours projected onto the $m_{\text {host }}-r_{e}$ plane for the de Vaucouleurs and exponential fits of the resolved sources.

\subsection{K Corrections and Filter Conversions}

Cousin's R band absolute magnitudes are presented for purposes of comparison with the low redshift BL Lacs in Urry et al. (2000). These corrections were performed by assuming SEDs for both the host galaxies and the nuclei. The models of Bruzual \& Charlot (1993) were used for the host galaxy SEDs, calibrated to the galaxy colours reported in Fukugita, Shimasaku \& Ichikawa (1995): a Hubble-type Ea spectrum $(B-R=1.57)$ for correcting the de Vaucouleurs fits, and a Hubble-type Sab $(B-R=1.34)$ for the exponential fits. A power law SED with $\alpha=-1$ was assumed for the BL Lac nuclei.

For the filter conversions, the redshifted SED was convolved with the transmission curve for the F $28 \times 50 \mathrm{LP}$ filter and then normalised to the best-fit host galaxy magnitudes. $\mathrm{K}$ corrections were performed assuming $H_{0}=70 \mathrm{~km} \mathrm{~s}^{-1} \mathrm{Mpc}^{-1}, \Omega_{M}=0.3, \Omega_{\lambda}=0.7$ (the cosmology used throughout).

The level of uncertainty in the host galaxy spectral corrections is potentially very large. For sources at these redshifts, the F28×50LP filter samples well below the restframe $4000 \AA$ break, and hence is highly sensitive to the star formation history of the galaxy. Uncertainties may be as large as 0.6 mag if the high-redshift host galaxies have colours closer to those of spiral galaxies, rather than the red colours observed at low redshift (Scarpa et al. 2000a), or even as large as 1 mag if they contain a strong starburst component. The quoted absolute magnitudes should be interpreted with this in mind.

Galactic extinction was corrected using the relation to HI column density from Shull \& Van Steenberg (1995): $E(B-V)=\log N(H I) /\left(21.72 \mathrm{~cm}^{-2} \mathrm{mag}^{-1}\right)$

\section{Host Galaxy Results}

The results of the fits for the single-component galaxy models and the PSF-only model are given in Table 2. Table 3 shows the results for the composite galaxy models. 


\subsection{Host Galaxy Brightnesses}

The average absolute R-band magnitude of the five resolved host galaxies - $0820+225$, 1144-379, 1249+174, 1422+580, and 1533+535 — for the de Vaucouleurs model fit, K corrected assuming an early-type spectrum, is $M_{R}=-24.3$ mag. This is much brighter than the average magnitude of $M_{R}=-22.85 \mathrm{mag}$ measured for the lower redshift BL Lac host galaxies in Urry et al. (2000). This strongly suggests that there has been significant luminosity evolution between the two samples, even taking into account the presence of the (high) upper limits in this sample. The average magnitude remains high even if a much bluer spectrum is used for $\mathrm{K}$ corrections: -23.7 mag for a Hubble-type Sab spectrum, and -23.51 mag for a 1 Gyr-old burst population.

\subsection{Host Galaxy Morphologies}

We were able to resolve complex morphologies in two cases. Both $0820+225$ and $1422+580$ clearly have bulge-dominated host galaxies. The effective radii derived from almost all of the de Vaucouleurs fits are very large, although the sizable errors on these radii encompass more sensible, smaller values. Nonetheless, the derived radii of $0820+225$ and $1533+535$ are unphysically large. These large scale sizes may indicate that some of the host galaxies in this sample may have substantial disk components as well as bulges. The bulge + disk host galaxy model is preferred with high significance for $0820+225$, and with marginal significance for $1422+580$ and $1533+535$.

Two of the unresolved sources, $0235+164$ and $1308+326$, show a significant amount of extended light which is fit well by an exponential disk component. This light can also be accounted for as resulting from an imperfectly subtracted sky, at the limit of the errors in the sky subtraction (and, in the case of $0235+164$, at the limit of the potential variation in the PSF). As such, the two are considered non-detections. However, many of the host galaxies show the same excess of extended light above the best-fit de Vaucouleurs profile. It is possible that these 'non-detections' are actually disky hosts on large scales. These sources would require longer integration times to confidently resolve the host galaxies.

\subsection{Close Environments and Evidence of Interactions}

Most of the BL Lacs in this sample appear to have close companions, nearby irregular structure, or both. $1533+535$ is the only source that has no such observable features. It is impossible to judge in the case of $0235+164$ because of the known intervening system. 
Discounting this BL Lac, five out of the remaining eight have apparent companions within a projected distance of $50 \mathrm{kpc}$, and four of the eight show signs of recent interactions, either companions within $10 \mathrm{kpc}$ or irregular structure.

This sample differs from the broader BL Lac sample of Urry et al. (2000), which exhibited close companions within $50 \mathrm{kpc}$ in $47 \%$ of cases, rather than the $62.5 \%$ as observed here. Very few of the broader sample showed signs of recent interactions, compared to $50 \%$ of this sample.

\subsection{Notes on Individual Objects}

0138-097 The host galaxy of this BL Lac appeared to be marginally resolved using the composite stellar PSF. With two of the alternate stellar PSFs, however, it produced fits which were not sufficiently better than the PSF-only fit. Thus, it is considered unresolved. The difficulty in resolving this host galaxy is compounded by the presence of a bright companion object $1^{\prime \prime} 44(10.5 \mathrm{kpc})$ to the southwest of the nucleus, and more structure within $5^{\prime \prime}$.

0235+164: The field of this object is complex, with bright structure extending to $\sim 3^{\prime \prime}$ $(\sim 30 \mathrm{kpc})$ from the nucleus. This structure has been well-studied in the past (e.g. Stickel et al. 1988; Yanni, York \& Gallagher 1989; Abraham et al. 1993; Falomo 1996), and is known to be an intervening system with redshift $z=0.524$. These STIS data resolve the complex structure in significantly more detail than previous observations, but we leave its analysis to a later work.

Fortunately the extended structure is concentrated to the south of the nucleus, leaving most of northern half clear for our host galaxy analysis. A host galaxy appeared resolved using the composite stellar PSF, however when an alternate stellar PSF was used and the background was over-subtracted to its uncertainty limit, it was not preferred over the PSFonly fit with sufficient confidence, and so is considered unresolved.

0820+225: The host galaxy of this source is the best resolved of the sample thanks to the relatively faint nucleus. Although the host has a strong bulge component, a model including both bulge and disk components provides a significantly better fit than the bulgeonly model if effective radius of the bulge is restricted to $\leq 20 \mathrm{kpc}$. A compact companion galaxy is also resolved at $\sim 0.7$ ( $5.5 \mathrm{kpc})$ to the west of the nucleus.

1144-379: This host galaxy is resolved, although not sufficiently well to distinguish between a bulge- or disk-dominated morphology. A very faint, arc-like wisp is resolved at $\sim 2^{\prime \prime} .25(18 \mathrm{kpc})$ to the northwest of the nucleus. 
1249+174: This host galaxy is definitely resolved; however, there was insufficient information to constrain its morphology. A bright, elongated structure is resolved extending from $\sim 0^{\prime \prime} 8$ to $\sim 1^{\prime \prime} 8$ ( $\sim 5$ to $13 \mathrm{kpc}$ ) to the northwest of the nucleus, while two compact objects are visible at $\sim 10^{\prime \prime}$ from the BL Lac.

1308+326: The host galaxy of this source is well fitted by an exponential disk profile. However, it cannot be confidently distinguished from the PSF if the background is oversubtracted to its uncertainty limit, and so it is considered unresolved. Two bright compact companion objects are within $6^{\prime \prime}(48 \mathrm{kpc})$ of the BL Lac, while a number of fainter objects are within half that distance.

1422+580: This host galaxy is well-resolved, and a de Vaucouleurs profile provides a significantly better fit than an exponential disk profile. A host galaxy model including both bulge and disk components provides a marginally better fit. The host galaxy appears smooth and is clearly elongated, with an axial ratio of 0.8. Several compact companion objects are in the near vicinity of the BL Lac, some faint ones within $\sim 1$ 1".5 (13 kpc).

1533+535: Another well-resolved, smooth-looking host galaxy, although in this case the morphology could not be constrained. This may be due to the presence of a disk component, as the best-fit de Vaucouleurs effective radius is unphysically large. A de Vaucouleurs + exponential profile gives a marginally better fit than the de Vaucouleurs-only model.

2240-260: The host galaxy of this source is unresolved. A few compact objects lie to the northeast, $\sim 6^{\prime \prime}(45 \mathrm{kpc}$ ) from the BL Lac.

\section{Host Galaxy Evolution}

To study the evolution of BL Lac host galaxies we combine the STIS observations described above (hereafter referred to as the high-z sample) with the lower-redshift BL Lacs observed in the WFPC2 snapshot survey (those with $z \leq 0.6$; hereafter the low-z sample). The results of the host galaxy analysis for these 63 low-z BL Lacs are listed in Table 4, and the details of the analysis described in Scarpa et al. (2000).

The STIS F28 $\times 50 \mathrm{LP}$ filter samples the rest-frame $\mathrm{U}$ to $\mathrm{V}$ bands at redshifts $z>0.6$, and so is particularly sensitive to star formation. This fact has enabled us to detect BL Lac host galaxies at greater distances than previously achieved. However, the star formation histories of the high-z BL Lacs are unknown, and thus the filter conversions and K corrections

are highly uncertain. Any measurement of luminosity evolution in absolute magnitude space would be subject to large errors. However, from NICMOS imaging of 10 low redshift BL Lacs 
(Scarpa et al. 2000a), we know that these host galaxies have colours consistent with old stellar populations. At the same time, conversion from F702W to $\mathrm{F} 28 \times 50 \mathrm{LP}$ magnitudes at lower redshifts is far less sensitive to star formation history than conversion from F28 $\times 50 \mathrm{LP}$ to F702W at higher redshifts. To minimise correction uncertainties we perform the analysis of host galaxy luminosity evolution using the measured F28 $\times 50 \mathrm{LP}$ apparent magnitudes of the high-redshift sample, corrected only for galactic extinction, and the derived F28 $\times 50 \mathrm{LP}$ apparent magnitudes of the low-redshift sources, converted from the measured F702W apparent magnitudes. The filter conversions used for the low-z sample are not fixed, but rather varied within the fitting process (described in $\$ 4.1$ ).

The host galaxy brightnesses of the combined sample are then compared to models of galaxy luminosity evolution. These models are derived using the GISSEL evolutionary synthesis models of Bruzual \& Charlot (1993) with a Salpeter IMF (Salpeter 1955), with the Padova 2000 library of isochrones (Girardi et al. 2000), and the BaSeL standard stellar library (Lejeune, Cuisinier \& Buser 1997).

Figure 5 shows the STIS F $28 \times 50$ LP Hubble diagram for the host galaxies, with the low-Z sample filter conversions performed assuming a solar metallicity burst population calibrated to match the typical early-type colour of $B-V=0.96$ (Fukugita, Shimasaku \& Ichikawa 1995). Also shown is the line for a non-evolving population with the same early-type spectrum (dashed line), and a non-evolving late-type spectrum (solar metallicity, age $=8$ Gyrs, star formation rate decay exponent $\tau=-3$ ) matching the Hubble type Sab colours of $B-V=0.57$ (Fukugita, Shimasaku \& Ichikawa 1995; dotted line). The high redshift host galaxies appear to be more luminous than predicted by the non-evolving models. However, the large error bars and the presence of non-detections in both the high-z and low-z samples necessitate rigorous testing.

\section{1. $\quad \chi^{2}$ Fitting with Censored Data}

The presence of host galaxy non-detections in some of the higher redshift objects means that the apparent luminosity evolution may just be a selection effect. Great care must be taken in dealing with the non-detections, and for this we employ survival analysis techniques.

The technique developed to apply survival analysis to the fitting of evolution models is derived from the EM algorithm (Dempster, Laird \& Rubin 1977). This algorithm uses maximum likelihood to estimate the regression coefficients of a 2-dimensional data set, and assumes that the censored data points have a normal distribution about the regression line. This regression line is determined iteratively, with the estimates for the weighting of the 
censored data points updated at each iteration. The assumption of a normal distribution makes the EM method less general than other, non-parametric methods, but given that the host galaxy luminosities have a roughly-normal distribution at low redshifts, this assumption seems reasonable.

As we are fitting the apparent magnitudes rather than the absolute magnitudes, we can't perform a simple regression analysis. Instead of iterating over regression line estimates, we iterate over evolution models. If we assume that each galaxy consists of a single, co-evolving population, then each model is defined by two parameters: magnitude zero point and either population age or formation redshift $\left(z_{\text {form }}\right)$ for the non-evolving and passively evolving models respectively.

Each iteration in population age or formation redshift implies a set of filter corrections for the low-z sample. Although there are only small differences in the filter correction between different galaxy models at low redshift, we wish to calculate $\chi^{2}$ given the measured F702W magnitudes, not the inferred F28 $550 \mathrm{LP}$ magnitudes, for each evolution model. With this in mind, we apply a new, self-consistent spectral correction for each new population age or formation redshift, rather than assume the same correction for all iterations. For the moment, we assume solar metallicity in all models.

\subsubsection{Single-Population Passive and Non-Evolving Models}

The passively evolving stellar population yielding the best fit to the F28 $\times 50 \mathrm{LP}$ apparent magnitudes of our sample has a formation redshift of $z_{\text {form }}=1.8$, with a reduced $\chi^{2}$ of 1.37 . The $1 \sigma$ uncertainties are $+0.7 /-0.3$ and the $3 \sigma$ uncertainties are $+1.0 /-0.5$. A better fit by a model with high formation redshift can be ruled out with high certainty $-z_{\text {form }}=3.0$

yields a reduced $\chi^{2}$ of 1.98 , while $z_{\text {form }}=5$ gives a reduced $\chi^{2}$ of 2.35 . An F-test (72 data points and 2 degrees of freedom) indicate a worse fit than the $z_{\text {form }}=1.8$ model with $95 \%$ and $99 \%$ significance respectively.

The data are also reasonably well fit by an extremely young non-evolving population. The best fit is for a population of age $0.02 \mathrm{Gyr}$, with reduced $\chi^{2}=1.51$ - a worse fit than the passive model, although without statistical significance. A population as young as 1 Gyr provides a poor fit to the data, with reduced $\chi^{2}=2.13$ : worse than the best passive model at the $97.5 \%$ significance level, and worse than the 0.02 Gyr model at the $95 \%$ significance level. A population of age 2.5 Gyrs gives a reduced $\chi^{2}$ of 2.38; worse than the passive model at the $99 \%$ level. Similar results are obtained for models of non-evolving late-type galaxy spectra with the same $B-V$ colours as the burst models. 
The young populations needed to fit the data do not agree with the colours observed in BL Lac host galaxies at low redshift. From Scarpa et al. (2000a), we know that these galaxies have $R-H=2.3 \pm 0.3$, consistent with an old stellar population. At the blue-most limit, these colours imply age $\sim 5$ Gyrs for a burst population. Fitting the normalization of a non-evolving model with age $=5$ Gyrs results in a reduced $\chi^{2}$ of 3.05. An F-test indicates that the passive evolution model gives a better fit than the 5 Gyr non-evolving model at the $99.9 \%$ significance level.

Figure 6 shows the Hubble diagrams with the best-fit passive and non-evolving models (top and middle). In each case, the low-z host galaxies are converted from $\mathrm{F} 702 \mathrm{~W}$ to F28 $\times 50$ LP magnitudes assuming the corrections implied by the evolution model plotted with a solid line. Visual inspection of the Hubble diagrams shows that the non-evolving model predicts galaxies about one magnitude too faint at $z>0.6$. Even much younger non-evolving models (1 Gyrs; middle, dashed line, fail to reproduce the high redshift colours, although an unphysically young model (0.02 Gyrs; middle, dotted line), does fit. Passive evolution models with high formation redshifts similarly underestimate the brightness of the $z>0.6$ host galaxies, as can be seen by the $z_{\text {form }}=5$ model (top, dashed line).

\subsubsection{Dual-Population Evolution Model}

While the above results indicate that the luminosity evolution is better fit by a model that includes a rapidly evolving stellar population, it may not be the entire population that is evolving. If an old stellar population is already in place, then a fresh burst of star formation involving only a fraction of the galaxy's mass may also reproduce the observed evolution. We fit the data with a model that comprised a passively evolving stellar population superimposed with a second population. This second population remains at constant (low) age down to a given redshift, after which it also evolves passively, to simulate a normally evolving galaxy with some degree of ongoing star formation, which dies out at some point. The model now includes five parameters: magnitude offset, formation redshift of the primary population $\left(z_{\text {form }}\right)$, the point at which the secondary population begins to evolve passively $\left(z_{*}\right)$, percentage mass of the secondary population relative to the primary, $m_{*}$, and the initial age of the secondary population, $a g e_{*}$.

A wide range of parameters provided fits of similar quality to the best-fit one-component model. For a primary population with a high formation redshift $\left(z_{\text {form }} \gtrsim 4\right)$, a secondary population of at least $1 \%$ of the mass of the primary (depending on $z_{*}$ and age $e_{*}$ ) produces reasonable fits. For a small secondary component of $1 \%$ to $3 \%, z_{*}$ can be as low as $\sim 0.5$ while still agreeing with the red colours observed in low-redshift BL Lac host galaxies. The best 
fit was found for $m_{*}$ of $2 \%, z_{*}=0.6, a g e_{*}=1 \mathrm{Gyr}$, and $z_{\text {form }}=5$, with reduced $\chi^{2}=1.29$.

The bottom panel of figure 6 shows the best-fit dual-population evolution model (solid line). The dashed line in this plot shows the evolution of the $z_{\text {form }}=5$ base population.

Although we can't distinguish between single- and dual-population models, we can say with high confidence that the observed luminosity evolution is significantly better represented by models which include star formation activity in the range $0.5<z \lesssim 2.5$, which diminishes rapidly or ceases altogether below $z \sim 0.5$, than it is by models in which the galaxies are undergoing steady star formation to low redshift, or by models in which all star formation activity had ceased by $z \sim 2.5$.

\subsection{Effects of Metallicity and Dust}

Given our knowledge of the red colours of low redshift BL Lac host galaxies, these data show conclusively that higher redshift BL Lac hosts are both significantly bluer and significantly brighter than their low redshift counterparts. This change is well modelled as evolution of the stellar population, however evolution in metallicity or the presence of dust may also play a part.

\subsubsection{Evolution in Metallicity}

The evolution results given so far are for populations with constant, solar metallicities. If the galaxies at higher redshift have much lower metallicities, the data can be fitted with older stellar populations. We simulate non-solar metallicities using the models of Westera et al. (2002).

Taking the host galaxies with $z>0.6$ to have extremely low metallicity - 0.02 solar and those with $z<0.6$ to have solar metallicity, the best-fit passively evolving population has $z_{\text {form }}=2.3{ }_{-0.7}^{+0.8}$ (1 1 errors), with reduced $\chi^{2}=1.28$, better at $97 \%$ confidence than a $z_{\text {form }}=5$ model (reduced $\chi^{2}=2.07$ ). The same metallicity evolution allows non-evolving populations to fit better. A population with age $=1$ Gyr gives reduced $\chi^{2}=1.99$, worse than the best-fit passive model with only $95 \%$ confidence (instead of $97.5 \%$ for a constant, solar metallicity). However this population still does not satisfy the low-redshift $R-H$ colour constraint.

Of course, a sudden transition from 0.02 solar to 1 solar metallicity at $z=0.6$ is not realistic; however, this scenario provides a limit on the extent to which metallicity evolution 
can affect the results.

\subsubsection{Effects of Dust Reddening on the Fits}

The presence of large amounts of dust in the host galaxies could potentially result in extreme reddening - enough to give a young population the $R-H$ colours observed in low redshift BL Lacs. However, there is a limit to how much dust can be added, as the preferential extinction caused by dust at shorter wavelengths makes the high-redshift population fainter, worsening the fit.

We applied models of dust extinction from Ferrara et al. (1999) to our spectral evolution models to determine whether a dust-reddened, non-evolving population could produce a reasonable fit to the data, with the constraint that the low-redshift $(z<0.4)$ host galaxies have $R-H=2.0$ (the bluest possible based on the NICMOS observations), after dust reddening. The extinction model which achieved this most efficiently was one based on the Milky Way extinction curve, assuming an elliptical galaxy geometry with a Jaffe density profile, and a central V-band optical depth of $\tau_{V}(0)=2$. With this model, a non-evolving, 1 Gyr-old population has $R-H=2$ at low redshift, and fits our Hubble diagram with reduced $\chi^{2}=2.21$. This is worse than the best-fit passive model at the $98 \%$ level by the F-test. To substantially improve this fit (worse only at the $95 \%$ level), a 0.6 Gyr population is needed, which, with this dust model, has $R-H=1.7$ - certainly far too blue for the low-redshift host galaxies.

Thus, no (non-evolving) dust model can make a non-evolving stellar population fit the data. The level of dust reddening needed even for these relatively poor fits, $\tau_{V}(0)=2$, is at the upper limit of dust optical depths estimated in ellipticals by Wise \& Silva (1996) or face-on spirals by Kuchinski et al. (1998), and is substantially higher that the limit predicted for ellipticals by Goudfrooij \& de Jong (1995) of $\tau_{V}(0)<0.7$, who find more typical values of $\tau_{V}(0) \sim 0.2-0.3$.

\subsubsection{Evolution in Dust Content}

It is possible to reproduce the observed luminosity evolution for a non- or weaklyevolving population if dust content is allowed to increase rapidly between $z \sim 0.8$ and $\sim 0.4$. Large changes at lower redshifts fail to fit the weak evolution seen at low-redshift, and extending the dust evolution to higher redshifts means that there is too much attenuation to reproduce the bright host galaxies at $z \gtrsim 0.6$. 
Assuming that the galaxies are relatively dust-free at $z \geq 0.8$, and that optical depth in dust increases linearly to $z=0.4$, the minimum final optical depth required to produce a reasonable fit to the data and to satisfy the low-z $R-H=2$ constraint is $\tau_{V}(0)=8$. This allows a non-evolving model as young as 0.8 Gyrs to fit the data with reduced $\chi^{2}=1.54$, within $1 \sigma$ of the best-fit passive evolution model.

Of course, $\tau_{V}(0)=8$ is extremely high - over an order of magnitude higher than the typical values determined for local ellipticals, and several times higher than those determined for star-forming galaxies (e.g. Calzetti 2001). If we assume a local optical depth of $\tau_{V}(0)=2$, the upper limit predicted for ellipticals, then the best-fit non-evolving evolution model gives reduced $\chi^{2}=2.01$ - still worse than the best passive model at the $\sim 95 \%$ level.

Approximately same level of dust evolution is required to make a passively evolving population with high formation redshift fit the data. A population with $z_{\text {form }}=5$ requires a local optical depth of $\tau_{V}(0)=10$ for reduced $\chi^{2}=1.52$, while the same population with $\tau_{V}(0)=2$ at $z=0$ gives reduced $\chi^{2}=2.09$ - worse than the $z_{\text {form }}=1.8$ model at $97 \%$ confidence.

A concurrent evolution in metallicity will allow these models to fit the data with a smaller increase in dust content. Using the metallicity evolution described above, the minimum local optical depth needed for a 2 Gyr-old non-evolving or a $z_{\text {form }}=5$ passively evolving population to fit the data (within $1 \sigma$ of the best passive model) is $\tau_{V}(0)=5$ (reduced $\chi^{2} \sim$ 1.5). This is still an extreme increase in dust content. If $\tau_{V}(0)=2$ locally, then these models are worse than the best passive model at the $\sim 95 \%$ confidence level.

Figure 7 shows the Hubble diagram with both the non-evolving and passive evolution models, dust and metallicity evolution included.

\subsection{Absolute Luminosity Evolution}

The lack of extended emission line light or a big blue bump has made it possible to use the extremely sensitive F28 ×50LP filter in this study. However, to compare our evolution results directly to other studies, it is necessary to determine this evolution in absolute nearinfrared magnitudes. This result depends strongly on the $\mathrm{K}$ corrections used. To find the full range of possible magnitude-redshift gradients, we perform regression analysis on absolute $\mathrm{K}$-band magnitudes using the range of $\mathrm{K}$ corrections implied by the full range of possible evolutionary models from the above analysis.

We use the EM algorithm to perform regression analysis while accounting for the cen- 
sored data points, via the IRAF task EMMETHOD. This task by itself does not take error bars into account. As the uncertainties in host galaxy magnitudes increase with redshift, they are an important consideration. To account for them, we use a Monte Carlo approach. One hundred simulated data sets were generated, randomizing each detected data point over a composite normal distribution, with the widths of the upper and lower halves of the distribution defined by the upper and lower error values for that data point. The regression coefficients and their uncertainties were determined for each of these 100 simulated data set with EM method, and the final coefficients taken to be the mean values. The uncertainty values were taken to be the mean of the individual uncertainties in the regression coefficients, added in quadrature with the standard deviation of the coefficients across the 100 simulations.

The steepest slope occurs when $\mathrm{K}$ corrections are derived from the evolutionary model with the oldest possible population at high redshift (at the $3 \sigma$ level, assuming no dust or metallicity evolution) - the passive evolution model with $z_{\text {form }}=2.8$. The shallowest slope is in the case of the youngest possible high-z population (also at the $3 \sigma$ level) - a passive model with $z_{\text {form }}=1.3$. Unsurprisingly, the best-fit passive evolution model, with $z_{\text {form }}=1.8$, gives a gradient about halfway between these. The results of the regression analysis using these three sets of $\mathrm{K}$ corrections are shown in Table 5.

A similar range of gradients are obtained if we assume a secondary burst component added to an older population, with a lower limit of $0.59 \mathrm{mag} / \mathrm{z}$ for a passive, old population plus a 0.1 Gyr burst involving 3\% of the galaxy's mass which itself passively evolves below $z=0.5$. For a constant-age, old population, or a population with high formation redshift we obtain $\sim 1.7 \mathrm{mag} / \mathrm{z}$. To obtain high redshift K-band magnitudes consistent with no K-band luminosity evolution, the stellar populations at $z>0.6$ must have ages less than 0.2 Gyrs. Such star formation histories can only be made to fit the observed Hubble diagram and satisfy the low-redshift colour constraints with extreme levels of dust evolution (from $\tau_{V}(0) \sim 0$ to $\tau_{V}(0) \sim 5$ between $z=0.8$ and 0.4$)$.

Using the best fit to the Hubble diagram to derive K corrections, and adding in quadrature the error in the regression analysis to the probable range of slopes from different $\mathrm{K}$ corrections, we determine the overall K-band luminosity evolution of BL Lac host galaxies to be $1.0 \pm 0.45 \mathrm{mag} / \mathrm{z}$. Figure 8 shows absolute K-band magnitude versus redshift, $\mathrm{K}$ corrected assuming a passively evolving population with $z_{\text {form }}=1.8$. Also plotted are the best-fit linear regression lines representing the magnitude-redshift gradients derived assuming the three different passive evolution models. 


\section{Discussion}

We detect luminosity evolution in BL Lac host galaxies, consistent with the primary stellar population evolving strongly from $1.5 \lesssim z \lesssim 2.5$, or with a smaller burst of star formation evolving from $z>0.5$, and inconsistent with a non-evolving population or a population evolving passively from high redshift. Interestingly, this formation epoch for the primary population corresponds roughly to the epoch of maximum quasar activity (e.g. Maloney \& Petrosian 1999; Shaver et al. 1999). This is consistent with a scenario in which black hole feeding and growth - i.e., the epoch of maximum AGN activity - coincides with the formation of the surrounding bulge (Kormendy \& Gebhardt 2001).

Radio galaxies (Djorgovski, Spinrad \& Marr 1985; Spinrad 1986; Spinrad \& Djorgovski 1987; Lilly \& Longair 1982; Lilly, Longair \& Allington-Smith 1985; Aragon-Salamanca et al. 1993; McLure \& Dunlop 2000) and the hosts of radio-loud quasars (Lehnert et al. 1999; Kukula et al. 2001; Hutchings 2001) exhibit similar evolution, probably dimming by of order 1 magnitude in $\mathrm{K}$ between $z=1$ and 0 . Although this evolution is not as well constrained, it is consistent with that seen in our sample. This is in agreement with the unified model of AGN in which radio galaxies, radio quasars and blazars are expected to have the same host galaxy properties. In contrast, BCGs (Aragon-Salamanca et al. 1993) other quiescent early-types (Lilly et al. 1995; Stanford et al. 1998; Bell et al. 2004) and radio-quiet quasar hosts (Kukula et al. 2001; Rix et al. 2001; Ridgway et al. 2001) exhibit flat, or even negative luminosity evolution, with local galaxies appearing as bright or brighter than those at $z \gtrsim 1$. Thus, the hosting of a powerful radio jet seems to be linked to a galaxy's luminosity evolution.

The luminosity evolution of BL Lac host galaxies is unambiguous, not affected by the biases inherent in other radio-loud classes (see $\S 1$ ). We can therefore ascribe the evolution observed in BL Lac hosts to a real change in galaxy luminosities, which in turn grants us more confidence that the evolution observed in other radio-loud hosts is real.

Furthermore, BL Lacs allow us significantly more confidence that the observed evolution is a luminosity evolution in individual host galaxies. There is mounting evidence to suggest that BL Lac objects are long-lived, possibly with lifespans on the same timescales as galaxy evolution. The most compelling is the flat, possibly negative number density evolution of many BL Lacs (Morris et al. 1991; Giommi, Menna \& Padovani 1999; Bade et al. 1998; Perlman et al. 1996). There are as many, if not more BL Lac objects now than during the epoch of peak quasar activity. The low accretion rates in BL Lacs (less than 1\% Eddington; O'Dowd, Urry \& Scarpa 2002; Wang, Staubert \& Ho 2002) also suggest that BL Lacs may burn stably for extended periods. If the jets of BL Lacs obtain most of their power from the rotational energy of the black hole (e.g. Blandford \& Zjanek 1977, Meier 2002), they are expected to have lifespans of the order of gigayears (Cavaliere \& Malquori 1999). Long-lived 
emission is also supported by their dynamically evolved state at low redshift (e.g., Urry et al. 2000) compared to the high frequency of interaction remnants and close companions at higher redshifts (see §3.3). Thus, with BL Lacs we may be observing luminosity evolution intrinsic to the host galaxies themselves, rather than evolution in the processes which select galaxies as hosts of radio-loud AGN.

Our results are consistent with and confirm the results of the ground-based study of Heidt et al. (2004), who find evidence of luminosity evolution in BL Lac host galaxies, although do not constrain this evolution. Of the sources which overlap the two samples $0820+225,1249+174,1422+580$ and $2240-260$ - Heidt et al. detect the host of $0820+225$. Our host galaxy magnitudes for this source agree within error, although our effective radii do not. We believed that this stems from the uncertainty and width of the ground-based PSF (of order that of the host itself in some cases).

\subsection{Possible Causes of Luminosity Evolution}

Both quiescent, early-type galaxies and radio-quiet AGN host galaxies are gaining luminous mass up to the present, in line with the predictions of hierarchical clustering models (Kauffmann \& Haehnelt 2000), therefore the luminosity of these galaxies does not change with time. This does not appear to be the case with the host galaxies of radio-loud AGN, whose dimming over cosmic time is apparently not offset by the accretion of significantly amounts of new material. A number of effects may contribute to this.

Evolution linked to emission processes: Low redshift radio-loud AGN used in evolution studies are dominated by sources with low accretion onto their SMBHs, such as the FR I and low-power FR II radio galaxies in the 3C sample (in which luminosity evolution has been best studied). These are also the proposed parent population of BL Lacs (Urry \& Padovani 1995). If a large fraction of these AGN are long-lived, jet-dominated sources, then we select against active recent merger histories when we observe them locally. Continued merger events following an AGN's formation epoch will refuel the nucleus after depletion of the initial supply of gas and possibly disrupt stable, jet-dominated emission (Cavaliere \& Padovani 1989), as well as add luminous mass to the galaxy, offsetting the dimming due to an aging primary stellar population.

Evolution Linked to Black Hole Properties: While the production of a radio jet is likely to be linked to the properties of the central SMBH, it is uncertain exactly which black hole properties are important in determining radio loudness. There is some evidence that high black hole mass plays a part (Franceschini, Vercellone \& Fabian 1998; Lacy et al. 2001; 
Nagar et al. 2002; Jarvis \& McLure 2002; O'Dowd, Urry \& Scarpa 2002; Dunlop et al. 2003), although this may just be a selection effect (Woo \& Urry 2002; Oshlack, Webster \& Whiting 2002; Ho 2002). Black hole spin seems the more likely candidate as the critical determinant (Meier 2002; O'Dowd et al. 2004). On the other hand, high host galaxy mass is expected to dampen radio emission (Bicknell 1995). Galaxies that have extreme SMBH properties relative to their mass should favor the production of radio jets. Such SMBHs may be expected to preferentially occur in those galaxies whose formation was completed by higher redshifts.

In the case of SMBH mass, hierarchical models (Kauffmann \& Haehnelt 2000) predict that earlier-formed galaxies will have higher SMBH-to-host galaxy mass ratios, due to the larger amounts of cold gas available for accretion at earlier epochs. A correlation has even

been observed between the fraction of a galaxy's mass in the black hole and the age of its stellar population (Merrifield et al. 2000).

In the case of spin, a black hole of a given mass that is formed from a small number of major merger events is likely to have a higher spin than one of the same mass formed from many less massive mergers, as the angular momenta of these lesser interactions will tend to cancel out unless they are correlated (Cavaliere \& Padovani 1989). Locally, this means black holes of given mass which formed during the epoch of major mergers, at $z \sim 1-2$, will have higher spin than whose whose formation proceeded to the present.

According to the scenarios outlined above, when we select radio sources at low redshift, we are dominated by low-accretion AGN with extreme SMBH properties that are preferentially produced at higher redshifts, and that should have had relatively quiescent merger histories since the epoch of formation of their central engines. We have more credible evidence that radio-loud AGN do exhibit just such merger histories, supporting the idea that the properties of radio-loud AGN SMBHs are typically defined at high redshift.

\subsection{The Merger History of Radio Hosts}

In $§ 3.3$ it was found that many of the BL Lacs in the high-z sample have either close companions $(62.5 \%)$ or signs of recent interactions $(50 \%)$. Fewer of the broader sample of Urry et al. (2000) had close companions $(47 \%)$ and only a handful $(<10 \%)$ showed signs of interactions. This supports the idea that the high-z BL Lacs are closer to their formation epoch, and that the observed luminosity evolution is linked to the merger history of these host galaxies.

It is impossible to perform a such a comparison over a similar redshift range for other 
radio-loud AGN, as flux limits induce a power-redshift correlation. Locally, however, FR I radio galaxies, which are thought to be the primary parent population of BL Lac objects, do show fewer signs of recent interactions than the more powerful FR IIs (Heckman et al. 1986; Smith \& Heckman 1989), with the latter hosted by bluer galaxies and showing a much higher incidence of distorted morphologies.

These observations, along with the scenarios outlined in $§ 5.1$, point to a scheme in which radio-loud AGN are formed in major mergers, typically at high redshift, while those observed locally have evolved from a significantly earlier formation epoch relatively free of further interactions.

Given this picture, it is expected that cluster environment will play a critical role in governing when and where radio-loud AGN form and survive. Formation will be dictated by a balance between a high density environment (clusters for merger frequency) and low cluster velocity dispersion (high redshift clusters or local field, to permit full mergers; Makino \& Ebisuzaki 1996). Survival as jet-dominated sources to low redshift will favour lower density environments, as we require fewer ongoing merger events. BL Lacs do prefer relatively poor cluster environments at low redshift $(z<0.4)$, of Abel richness class $\sim 0$ to 1 (Falomo, Pesce \& Treves 1993; Pesce, Falomo \& Treves 1994; Fried, Stickel \& Kühr 1993; Wurtz et al. 1997; Smith, O’Dea \& Baum 1995). At higher redshifts, their environments are not well known, although at $z>0.6$ at least some are found in rich clusters (Fried, Stickel \& Kühr 1993; Wurtz et al. 1997). This is consistent with the picture in which BL Lacs are preferentially produced in cluster environments at high redshift (when cluster velocity dispersions were lower), but survive only in the sparcer regions of these clusters. Reports on the environmental evolution of other classes of radio-loud AGN are conflicting, as are the environmental differences between radio-loud and radio-quiet AGN.

\section{Conclusions}

Nine BL Lac objects with $z>0.6$ were imaged with HST, using the STIS CCD and the F28 $\times 50$ LP filter. The host galaxies of five of these were resolved with high confidence. For all but $0820+225$, these are the first detections of host galaxies in these sources, and include the two highest redshift BL Lac host galaxies detected to date. These galaxies are bright, with an average absolute magnitude of $\left\langle\mathrm{M}_{R}>=-24.3\right.$ mag assuming an early-type spectrum and -23.7 mag assuming a late-type spectrum.

The morphologies of two of the galaxies were well resolved. Both $1422+580$ and $0820+225$

are better fit by a de Vaucouleurs profile than by an exponential disk profile, indicating bulge 
dominated hosts, as is typical for radio-loud AGN. However, the host galaxy of $0820+225$ was significantly better fit by a composite model including both bulge and disk components than by a bulge component alone. Many, including some of those technically considered non-detections, show evidence of having extended, disky components. A large fraction of the sample show evidence of close companions and distorted morphologies — far more than are observed at lower redshift.

Combining the derived host galaxy magnitudes with the low redshift host galaxies from Urry et al. (2000), we measure luminosity evolution in a sample of 72 HST-imaged BL Lac host galaxies spanning the range $0 \lesssim z \lesssim 1$. We find that the Hubble diagram is well fit by a model of a stellar population evolving passively from $z=1.8_{-0.3}^{+0.7}$. Alternatively, an older population with a small fraction $(\lesssim 3 \%)$ undergoing active star formation to $z \sim 0.5$ also fits the data well. A single population undergoing passive evolution from a high formation redshift $\left(z_{\text {form }} \gtrsim 5\right)$ can be ruled out with high confidence. By constraining the colours of the low redshift host galaxies based on NICMOS observations, models including no luminosity evolution can also be ruled out with high confidence. Non-evolving and high-z $\mathrm{z}_{\text {form }}$ passive evolution models are still ruled out when reasonable models of dust and metallicity evolution are included.

By K-correcting the host galaxy magnitudes based on the range of acceptable fits to the Hubble diagram, we determined an average K-band luminosity evolution of $1.0 \pm 0.45$ magnitudes per unit redshift.

The fact that flux selection of BL Lacs is dominated by beaming angle rather than intrinsic brightness, together with the low levels of extended emission from ionized gas and scattered nucleur light in these sources, mean that they are relatively free of the biases affecting quasar studies. This increases our confidence that the observed luminosity evolution is real, and in turn in the luminosity evolution observed in other classes of radio-loud AGN. We also have reason to believe that the observed evolution is intrinsic to the galaxies themselves, as evidence suggests that BL Lacs may have lifespans comparable to the timescale for galaxy evolution.

The luminosity evolution of BL Lac host galaxies is steeper than that observed in radioquiet host galaxies, brightest cluster galaxies, and quiescent early-type galaxies, and is consistent with that observed in other radio-loud hosts. The absence of luminosity evolution in radio-quiet and quiescent galaxies suggests that dimming due to an aging population is offset by the accretion of new material, as is predicted by hierarchical clustering models. This appears not to be the case in the host galaxies of radio-loud sources, which evolve strongly from some redshift greater than $z \sim 0.5$, but also must have experienced significant star formation activity in the range $0.5<z<2.5$. This is further supported by the fact that BL Lacs with 
$z>0.6$ are much more frequently in interacting systems, and have more close companions than those at low redshift, which appear dynamically evolved. These observations lead to the following conclusions:

First, it seems that the physical conditions that allow a galaxy to generate a radio jet are preferentially produced in a particular cosmic epoch, albeit a broad one: $0.5<z<2.5$. This may be linked to the amount of gas available for fueling, which is also believed to define the quasar epoch. However, to explain the radio-loud-radio-quiet dichotomy, it must also be linked to the production of the right SMBH conditions.

Second, radio-loud AGN hosts at low redshift accrete less new material after the epoch in which they seemingly form their SMBHs than do other early-type galaxies. A number of effects may result in the selection of inactive recent merger histories when we select radioloud AGN at low redshift; however, all point to a scenario in which many radio-loud AGN observed at low redshift have survived from a much earlier formation epoch.

The evolution observed in the stellar populations of radio-loud AGN suggests the black holes which power these radio sources are born in gas-rich interactions at $z>0.5$. At low redshift, where samples are dominated by low-accretion sources, these observations suggest that we are observing the last embers of the radio quasar epoch - old AGN with massive, rapidly-rotating black holes which have experienced little merger activity since the formation of their central engines.

Support for this work was provided by NASA through grant numbers HST-GO-09121.01A and HST-GO-09223.01-A from the Space Telescope Science Institute, which is operated by AURA, Inc., under NASA contract NAS5-26555. 


\section{REFERENCES}

Abraham R. C., Crawford, C. S., Merrifield, M. R., Hutchings, J. B., \& Hardy, I. M. 1993, ApJ 415, 101

Aragon-Salamanca A., Ellis, R. S., Couch, W. J., \& Carter, D. 1993 MNRAS 262, 764

Bade N., Beckmann, V., Douglas, N. G., Barthel, P. D., Engels, D., Cordis, L., Nass, P., \& Voges, W. 1998 AA 334,459

Barth, A. J., Sarzi, M., Rix, H.-W., Ho, L. C., Filippenko, A. V., \& Sargent, W. L. W. 2001 ApJ 555, 685

Barthel, P. 2001 New Astronomy Reviews 45, 591

Bell, E., Wolf, C., Meisenheimer, K., et al., 2004 STScI May Symposium, Baltimore, MD

Bicknell, G. V. 1995 ApJS 101, 29

Blandford, R. D. \& Znajek, R. L. 1977 MNRAS 179, 433

Bower, G. A., Green, R. F., Bender, R., et al. 2001 ApJ 550, 75

Boyle B. J., Shanks, T., \& Peterson, B. A. 1988 MNRAS 235, 935

Bruzual, G. \& Charlot, S. 1993 ApJ 405, 538

Calzetti, D. 2001 PASP 113, 1449

Canalizo, G. \& Stockton, A. 2001 ApJ 555, 719

Cavaliere, A. \& Padovani, P. 1989 ApJ 340, L5

Cavaliere, A. \& Malquori, D. 1999 ApJ 516, L9

Connolly, A. J., Szalay, A. S., Dickinson, M., Subbarao, M. U., Brunner, R. J. 1997 ApJ 486 L11

Cowie, L. L., Songaila, A., Hu, E.r M., \& Cohen, J. G. 1996 AJ 112, 839

Cowie, L. L., Barger, A. J., Bautz, M. W., Brandt, W. N., \& Garmire, G. P. 2003 ApJ 584, L57

Dempster, A. P., Laird, N. M., \& Rubin, D. B. 1977 J. Roy. Statist. Soc. B 39, 1

de Young, D. S. 1989 ApJ 342 L59

Di Matteo, T., Croft, R. A. C., Springel, V., \& Hernquist, L. 2003 ApJ 593, 56

Djorgovski, S., Spinrad, H., \& Marr, J. 1985 in Lect. Notes Phys. Vol. 232, New aspects of galaxy photometry, ed. J. L. Nieto, (Berlin: Springer-Verlag), p. 193

Driver, S. P., Windhorst, Rogier A., \& Griffiths, R. E. 1995 ApJ 453, 48

Dunlop, J. S. \& Peacock, J. A. 1993 MNRAS 263, 936 
Dunlop, J. S., McLure, R. J., Kukula, M. J., Baum, S. A., O’Dea, C.P., \& Hughes, D. H. 2003 MNRAS 340, 1095

Falomo, R., Pesce, J. E., \& Treves, A. 1994 ApJ 411, L63

Falomo, R. 1996 MNRAS 283, 241

Ferrara, A., Bianchi, S., Cimatti, A. \& Giovanardi, C. 1999 ApJS 123, 437

Franceschini, A., Vercellone, S. \& Fabian, A. C. 1998 MNRAS 310, L5

Franceschini, A., Hasinger, G., Miyaji, T, \& Malquori, D. 1999 MNRAS 310, L5

Fried, J. W., Stickel, M., \& Kühr, H. 1993 A\&A 268, 53

Fukugita, M., Shimasaku, K. \& Ichikawa, T. 1995, PASP, 107, 945

Gebhardt, K., Richstone, D., Tremaine, S., et al. 2003 ApJ, 583, 92

Giommi, P., Menna, M. T. \& Padovani, P 1999 MNRAS 310, 465

Girardi, L., Bressan, A., Bertelli, G. \& Chiosi, C. 2000, A\&AS, 141, 371.

Goudfrooij, P. \& de Jong, T. 1995 A\&A 298, 784

Gonzalez Delgado, R. M. 1995 PASP 107, 1130

Haehnelt, M. G. \& Rees, M. J. 1993 MNRAS 263, 168

Hasinger, G. 2003 in XEUS - studying the evolution of the hot universe, Ed. G. Hasinger, Th. Boller, \& A.N. Parmer, MPE Report 281, p. 7

Heckman, T. M., Bothun, C. D., Balick, B., \& Smith, E. P. 1984 AJ 89, 958

Heckman, T. M., Smith, E. P., Baum, S. A., van Breugel, W. J. M., Miley, G. K., Illingworth, G. D., Bothun, G. D., \& Balick, B. 1986 ApJ 311, 526

Heisler, C. A. $1991 \mathrm{PhD}$ Thesis

Heidt, J., Trölle, M., Nilsson, K., Jäger, K., Takalo, L., Rekola, R., \& Sillanpää, A. 2004 A\&A 418, 8131991 $\mathrm{PhD}$ Thesis

Ho, L. C. 2002 ApJ 564, 120

Hooper, E. J., Impey, C. D. \& Foltz, C. B. 1997 ApJ 480, L95

Hutchings, J. B. \& Campbell, B. 1983 Nature 303, 584

Hutchings J. B., Crampton, D., \& Campbell, B. 1984 ApJ 280, 41

Hutchings, J. B. \& Neff, S. G. 1992 AJ 104, 1 
Hutchings, J. B. 2001 in QSO Hosts and their Environments, ed. I. Márquez, J. Masegosa, A. del Olmo, L. Lara, E. Garciá, \& J. Molina, (Dordrecht: Kluwer), p. 71

Jarvis, M. J. \& McLure, R. J. 2002 MNRAS 336, L38

Joseph, C. L., Merritt, D., Olling, R., et al. 2001 ApJ 550, 668

Kauffmann, G., Charlot, S., \& White, S. D. M. 1996 MNRAS 283, 117

Kauffmann, G. \& Haehnelt, M. G. 2000 MNRAS 311, 576

Kormendy, J. \& Gebhardt, K. 2001 in The 20th Texas Symposium on Relativistic Astrophysics, ed H. Martel \& J. C. Wheeler (AIP)

Kuchinski, L. E., Terndrup, D. M., Gordon, K. D.\& Witt, A. N. 1998 AJ 115, 1438

Kukula, M. J., Dunlop, J. S., McLure, R. J., Miller, L., Percival. W. J., Baum, S. A., \& O'Dea, C. P. 2001 MNRAS 326, 1533

Lacy, M., Laurent-Meuleisen, S. A., Ridgway, S. E., Becker, R. H., \& White, R. L. 2001 ApJ 551, L17

Lehnert, M. D., van Breugel, W. J. M., Heckman, T. M., \& Miley, G. K. 1999 ApJS 124, L11

Lejeune, Th., Cuisinier, F., \& Buser, R. 1997, A\&AS, 125, 229

Lilly, S. J. \& Longair, M. S., 1982 MNRAS 199, 1053

Lilly, S. J., Longair, M. S., \& Allington-Smith, J. R. 1985 MNRAS 215, 37L

Lilly, S. J., Tresse, L., Hammer, F., Crampton, D, \& Le Fevre, O. 1995 ApJ 455, L108

Madau, P., Ferguson, H. C., Dickinson, M. E., Giavalisco, M., Steidel, C. C., \& Fruchter, A. 1996 MNRAS 283,1388

Magorrian, J., Tremaine, S., Richstone, D. et al. 1998 AJ 115, 228

Makino, J. \& Ebisuzaki, T. 1996 ApJ 465, 527

Maloney, A. \& Petrosian, V. 1999 ApJ 518, 32

McLeod, K. K., Rieke, G. H. \& Storrie-Lombardi, L. J. 1999 ApJ 511, L67

McLure, R. J. \& Dunlop, J. S. 2000 MNRAS 317, 249

Meier, D. L. 2002 NewAR 46, 247

Merrifield, M. R., Forbes, Duncan A., \& Terlevich, A. I. 2000 MNRAS 313, L29

Morris, S. L., Stocke, J. T., Gioia, I. M., Schild, R. E., Wolter, A., Maccacaro, T., \& Della Ceca, R. 1991 ApJ 380, 49

Nagar, N. M., Falcke, H., Wilson, A. S., \& Ulvestad, J. S. 2002 AA 392, 53 
O’Dowd, M., Urry, C. M., \& Scarpa, R. 2002 ApJ 580, 96

O’Dowd, M., Urry, C. M., Scarpa, R., Wayth, R.., \& Webster, R. L. 2004 ApJ, submitted

Oshlack, A. Y. K. N., Webster, R. L., \& Whiting, M. T. 2002 ApJ 576, 81

Perlman, E. S., Stocke, J. T., Wang, Q. D., \& Morris, S. L. 1996 ApJ 456, 451

Pesce, J. E., Falomo, R., \& Treves, A. 1994 AJ 107, 494

Rees, M. 1989 MNRAS 239, 1

Ridgway, S. E., Heckman, T. M., Calzetti, D., \& Lehnert, M. 2001 ApJ 550, 122

Rix, H.-W., Falco, E. E., Impey, C., Kochanek, C., Lehr, J., McLeod, B., Muoz, J., \& Peng, C. 2001 in ASP Conf. Series 237, Gravitational Lensing: Recent Progress and Future Go, ed. T. G. Brainerd \& C. S. Kochanek, (San Francisco: ASP), p. 169

Salpeter, E. E. 1955 ApJ, 121, 161

Sarzi, M., Rix, H.-W., Shields, J. C. et al. 2001 ApJ 550, 65

Scarpa, R., Urry, C. M., Falomo, R., Pesce, J. \& Treves, A. 2000 ApJ 532, 740

Scarpa, R., Urry, C. M., Padovani, P., Calzetti, D., O’Dowd, M. 2000 ApJ 544, 258

Scarpa, R. \& Urry, C. M. 2001 ApJ 556, 749

Schade, D. J., Boyle, B. J. \& Letawsky, M. 2000 MNRAS, 315 \& 498

Schmidt, M. \& Green R. F. 1983 ApJ 269, 352

Silk. J. \& Rees, M. J. 1998 AA 331, L1

Shaver, P. A., Wall, J. V., Kellermann, K. I., Jackson, C. A., \& Hawkins, M. R. S. 1996 Nature 384, 439

Shaver, P. A., Hook, I. M., Jackson, C. A., Wall, J. V., \& Kellermann, K. I. 1999 in ASP Conf. Series 156, High Redshift Radio Lines, (San Francisco: ASP), p. 163

Shull \& Van Steenberg 1995 ApJ 294, 599

Smith, E. P., Heckman, T. M., Bothun, G. D., Romanishin, W. \& Balick, B. 1986 ApJ 306, 64

Smith, E. P. \& Heckman, T. M. 1989 ApJ 341, 658

Smith, E. P., O’Dea, C. P., \& Baum, S. A. 1995 ApJ 441, 113

Spinrad, H. 1986 PASP 98, 269

Spinrad, H. \& Djorgovski, S. 1987 in Proc. IAU Symp. 124, Observational Cosmology, ed. A. Hewitt, G. Burbidge, L.-Z. Fang, (Dordrecht: Reidel), p. 129 
Stanford, S. A., Eisenhardt, P. R., \& Dickinson, M. 1998 ApJ 492, 461

Stickel, M. Fried, J. W., \& Kühr, H. 1988 A\&A 198, L13

Urry, C. M. \& Padovani, P. 1995, PASP 107, 803

Urry, C. M., Scarpa, R., O’Dowd, M., et al. 2000, ApJ 532, 816

van der Marel, R. P. 1999, AJ 117, 744

Veŕon-Cetty, M.-P \& Woltjer, L. 1990 A\&A 236, 69

Wang, Y. P., Biermann, P. L., \& Wandel, A. 2000 A\&A 361, 550

Wang, J.-M., Staubert, R, \& Ho, L., C. 2002 ApJ 579, 554

Westera, P., Lejeune, T., Buser, R., Cuisinier, F. \& Bruzual, G. 2002, A\&A, 381, 524

Whittle M. 1992 ApJ 387, 121

Wise, M. W. \& Silva, D. R. 1996 ApJ 461, 155

Woo, J.-H. \& Urry, C. M. 2002 ApJ 581, L5

Wurtz, R., Stocke, J. T., Ellingson, E., \& Yee, H. K. C. 1997 ApJ 480, 547

Yanni, B., York, D. G., \& Gallagher, J. S. 1989 ApJ 338, 735

Yee, H. K. C. 1987 AJ 94, 1461

Zirm, A. W., Dickinson, M. \& Dey, A. 2003 ApJ 585, 90 
Table 1.

\begin{tabular}{cccccccc}
\hline \hline Name & z & Type $^{\mathrm{a}}$ & $m_{R}$ (nucl. $^{\mathrm{b}}$ & $M_{R}(\text { nucl. })^{\mathrm{c}}$ & $m_{R}(\text { host })^{\mathrm{d}}$ & Exp. Time (s) & Obs. Date) \\
\hline $0138-097$ & 0.733 & $\mathrm{~L}$ & 17.7 & -25.57 & $>19.57$ & 2554.0 & $24 / 07 / 2001$ \\
$0235+164$ & 0.94 & $\mathrm{~L}$ & 18.90 & -25.03 & $>19.4$ & 5429.0 & $18 / 07 / 2001$ \\
$0820+225$ & 0.951 & $\mathrm{~L}$ & 20.0 & -23.97 & $>21.25$ & 5473.0 & $17 / 09 / 2001$ \\
$1144-379$ & 1.048 & $\mathrm{~L}$ & 18.0 & -26.22 & $>22.72$ & 5615.0 & $04 / 08 / 2001$ \\
$1249+174$ & 0.644 & $\mathrm{H}$ & 18.5 & -24.43 & $>21.3$ & 2552.0 & $21 / 07 / 2001$ \\
$1308+326$ & 0.997 & $\mathrm{~L}$ & 18.1 & -25.99 & $>20.13$ & 5551.0 & $22 / 07 / 2001$ \\
$1422+580$ & 0.683 & $\mathrm{H}$ & 19.07 & -24.01 & $>21.7$ & 4644.0 & $26 / 08 / 2001$ \\
$1533+535$ & 0.89 & $\mathrm{H}$ & 18.60 & -25.19 & $>19.45$ & 4861.0 & $14 / 09 / 2001$ \\
$2240-260$ & 0.774 & $\mathrm{~L}$ & 17.5 & -25.91 & $>21.45$ & 2578.0 & $27 / 07 / 2001$ \\
\hline
\end{tabular}

aSED type: $\mathrm{H}=\mathrm{HBL}\left(F_{1 k e V} / F_{5 G H z}>5.5\right)$ and $\mathrm{L}=\mathrm{LBL}\left(F_{1 k e V} / F_{5 G H z}<5.5\right)$

bApparent R-band magnitude of source, not extinction or K corrected.

${ }^{\mathrm{c}}$ Absolute R-band magnitude of source, extinction corrected and $\mathrm{K}$ corrected assuming $\alpha=-1$, $H_{0}=70 \mathrm{~km} \mathrm{~s}^{-1} \mathrm{Mpc}^{-1}, \Omega_{M}=0.3, \Omega_{\lambda}=0.7$.

dimit on apparent R-magnitude of the host galaxy from Urry et al. (2000). 
Table 2. Results of two-component host galaxy fits

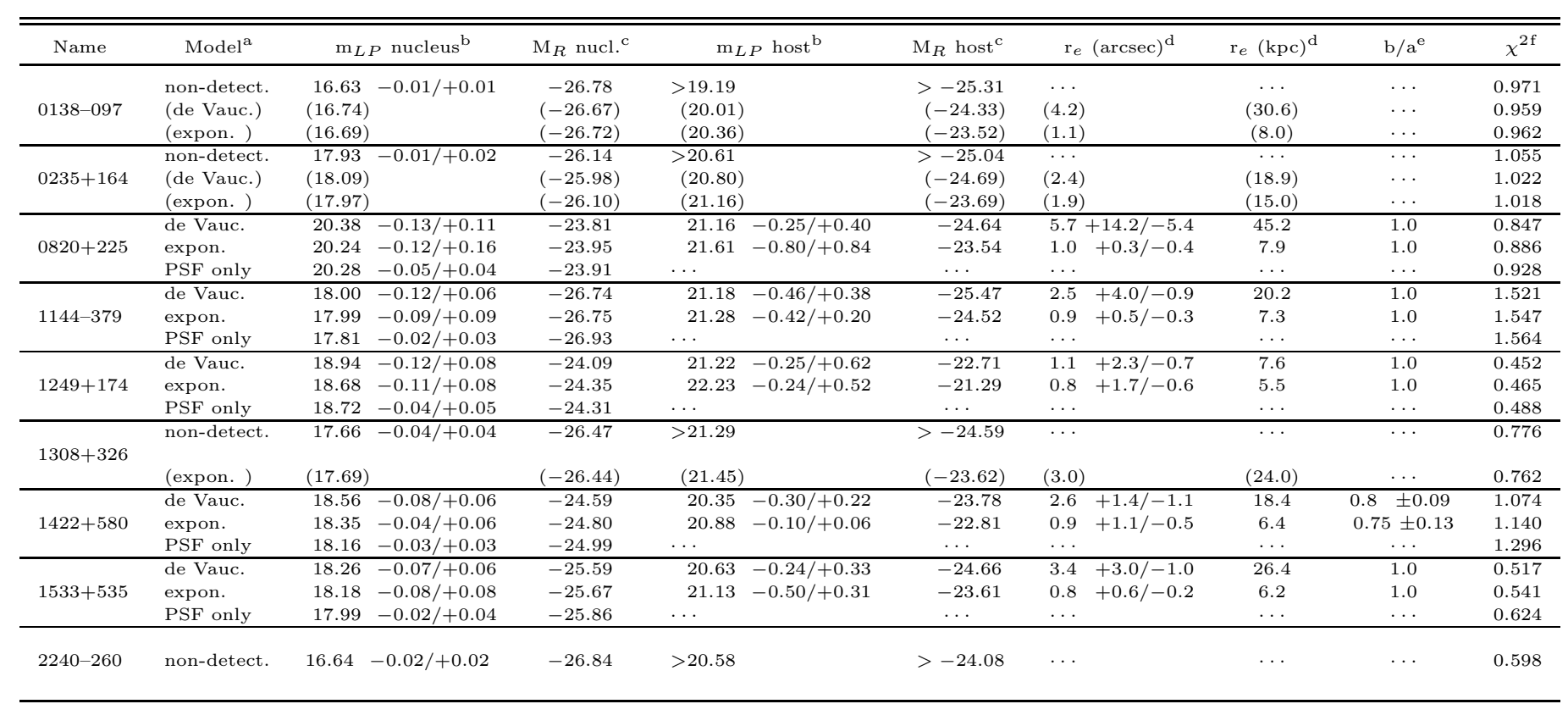

${ }^{a}$ Model fitted: PSF+de Vaucouleurs, PSF+exponential disk, or PSF only; non-detect indicates fit with PSF alone where host galaxy is not resolved. Bracketed values indicate best host galaxy fits, although are technically non-detections.

${ }^{\mathrm{b}}$ Apparent magnitude of nucleus/host galaxy in STIS F28 $\times 50 \mathrm{LP}$ band, not extinction corrected or $\mathrm{K}$ corrected.

${ }^{\mathrm{c}}$ Absolute magnitude of nucleus/host galaxy in Cousins $\mathrm{R}$ band, corrected for galactic extinction and $\mathrm{K}$ corrected assuming $\alpha=1$ power law for nuclei, early-type spectrum for de Vaucouleurs hosts and late-type spectrum for exponential disk hosts, and $H_{0}=70 \mathrm{~km} \mathrm{~s}^{-1} \mathrm{Mpc}^{-1}, \Omega_{M}=0.3, \Omega_{\lambda}=0.7$.

${ }^{\mathrm{d}}$ Characteristic radius, assuming $q_{0}=0$ for kpc radii.

eAxial ratio.

${ }_{\mathrm{f}}^{\mathrm{f}}$ Reduced $\chi^{2}$ for fit, calculated comparing full two-dimensional model to the image. 
Table 3. Results of three-component host galaxy fits

\begin{tabular}{|c|c|c|c|c|c|c|c|c|}
\hline Name & $M_{R}(\text { nucl. })^{\mathrm{a}}$ & $m_{R}(\text { nucl. })^{\mathrm{b}}$ & Component $^{\mathrm{c}}$ & $\mathrm{m}_{L P}(\text { host })^{\mathrm{d}}$ & $\mathrm{M}_{R}(\text { host })^{\mathrm{e}}$ & $\mathrm{r}_{e}\left({ }^{\prime \prime}\right)^{\mathrm{f}}$ & $\mathrm{r}_{e}(\mathrm{kpc})^{\mathrm{g}}$ & $\chi^{2 \mathrm{~h}}$ \\
\hline \multirow{2}{*}{$0820+225$} & \multirow[t]{2}{*}{20.37} & \multirow[t]{2}{*}{-25.14} & \multirow{2}{*}{$\begin{array}{c}\text { de Vauc. } \\
\text { expon. }\end{array}$} & 22.18 & -24.85 & 1.4 & 11.0 & \multirow[t]{2}{*}{0.811} \\
\hline & & & & 21.63 & -24.75 & 2.0 & 15.8 & \\
\hline \multirow[t]{2}{*}{$1144-379$} & \multirow[t]{2}{*}{17.98} & \multirow[t]{2}{*}{-28.12} & \multirow{2}{*}{$\begin{array}{c}\text { de Vauc. } \\
\text { expon. }\end{array}$} & 21.35 & -26.28 & 1.1 & 8.9 & \multirow[t]{2}{*}{1.51} \\
\hline & & & & 21.90 & -24.88 & 1.3 & 10.5 & \\
\hline \multirow{2}{*}{$1249+174$} & \multirow[t]{2}{*}{18.71} & \multirow{2}{*}{-25.54} & \multirow{2}{*}{$\begin{array}{c}\text { de Vauc. } \\
\text { expon. }\end{array}$} & 21.91 & -23.37 & 0.7 & 4.8 & \multirow[t]{2}{*}{0.446} \\
\hline & & & & 22.79 & -22.08 & 1.9 & 13.1 & \\
\hline \multirow[t]{2}{*}{$1422+580$} & \multirow[t]{2}{*}{18.37} & \multirow[t]{2}{*}{-26.02} & \multirow{2}{*}{$\begin{array}{l}\text { de Vauc. } \\
\text { expon. }\end{array}$} & 21.00 & -24.82 & 1.4 & 9.9 & \multirow[t]{2}{*}{1.049} \\
\hline & & & & 21.33 & -24.05 & 2.4 & 17.0 & \\
\hline \multirow[t]{2}{*}{$1533+535$} & \multirow[t]{2}{*}{18.25} & \multirow[t]{2}{*}{-26.90} & \multirow{2}{*}{$\begin{array}{c}\text { de Vauc. } \\
\text { expon. }\end{array}$} & 21.10 & -25.52 & 1.8 & 14.0 & \multirow[t]{2}{*}{0.505} \\
\hline & & & & 21.59 & -24.47 & 3.0 & 23.3 & \\
\hline
\end{tabular}

aApparent magnitude of nucleus in STIS F28×50LP band, not extinction or K corrected.

${ }^{\mathrm{b}}$ Absolute R-band magnitude of nucleus, corrected for galactic extinction and $\mathrm{K}$ corrected assuming $\alpha=1$, $H_{0}=70 \mathrm{~km} \mathrm{~s}^{-1} \mathrm{Mpc}^{-1}, \Omega_{M}=0.3, \Omega_{\lambda}=0.7$.

${ }^{\mathrm{c}}$ de Vauc. = de Vaucouleurs (bulge) component; expon. = exponential disk component.

${ }^{\mathrm{d}}$ Apparent magnitude of host galaxy in F28 $\times 50 \mathrm{LP}$ band, not extinction or K corrected.

${ }^{\mathrm{e}}$ Absolute R-band magnitude of host galaxy, extinction corrected and K corrected assuming early- and late-type spectra for de Vaucouleurs and exponential components respectively, with $H_{0}=70 \mathrm{~km} \mathrm{~s}^{-1} \mathrm{Mpc}^{-1}, \Omega_{M}=0.3$, $\Omega_{\lambda}=0.7$.

${ }^{\mathrm{f}}$ Characteristic radius in arcseconds.

${ }^{\mathrm{g}}$ Characteristic radius in kiloparsecs, assuming $q_{0}=0$.

${ }^{\mathrm{h}}$ Reduced $\chi^{2}$ for fit, calculated comparing full two-dimensional model to the image. 
Table 4. The low-z sample: BL Lac host galaxies from Urry et al. (2000).

\begin{tabular}{|c|c|c|c|c|c|c|c|c|}
\hline Name & $\mathrm{z}$ & Type $^{\mathrm{a}}$ & $\mathrm{m}_{R}$ nucleus $^{\mathrm{b}}$ & $\mathrm{m}_{R} \operatorname{host}^{\mathrm{c}}$ & $\mathrm{m}_{R}$ nucl. ${ }^{\mathrm{d}}$ & $\mathrm{m}_{R}$ host $^{\mathrm{e}}$ & $\mathrm{r}_{e}(\operatorname{arcsec})^{\mathrm{f}}$ & $\mathrm{r}_{e}(\mathrm{kpc})^{\mathrm{g}}$ \\
\hline $0118-272$ & 0.559 & $\mathrm{~L}$ & $15.78 \pm 0.10$ & $>19.09$ & -26.78 & $>-23.47$ & - & 0.0 \\
\hline $0122+090$ & 0.339 & $\mathrm{H}$ & $21.98 \pm 0.25$ & $18.88 \pm 0.04$ & -19.29 & -22.85 & $1.05 \pm 0.10$ & 5.08 \\
\hline $0158+001$ & 0.229 & $\mathrm{H}$ & $18.38 \pm 0.06$ & $18.27 \pm 0.03$ & -21.91 & -22.29 & $1.90 \pm 0.10$ & 6.96 \\
\hline $0229+200$ & 0.139 & $\mathrm{H}$ & $18.58 \pm 0.35$ & $15.85 \pm 0.01$ & -20.52 & -23.41 & $3.25 \pm 0.07$ & 7.97 \\
\hline $0257+342$ & 0.247 & $\mathrm{H}$ & $19.18 \pm 0.30$ & $17.93 \pm 0.01$ & -21.30 & -22.85 & $1.75 \pm 0.12$ & 6.78 \\
\hline $0317+183$ & 0.190 & $\mathrm{H}$ & $18.28 \pm 0.05$ & $17.59 \pm 0.01$ & -21.56 & -22.46 & $3.25 \pm 0.10$ & 10.31 \\
\hline 0331-362 & 0.308 & $\mathrm{H}$ & $19.03 \pm 0.10$ & $17.81 \pm 0.02$ & -22.00 & -23.62 & $3.10 \pm 0.20$ & 14.07 \\
\hline $0347-121$ & 0.188 & $\mathrm{H}$ & $18.28 \pm 0.15$ & $17.72 \pm 0.01$ & -21.53 & -22.30 & $1.25 \pm 0.05$ & 3.93 \\
\hline 0350-371 & 0.165 & $\mathrm{H}$ & $18.03 \pm 0.15$ & $17.08 \pm 0.01$ & -21.46 & -22.60 & $1.70 \pm 0.07$ & 4.81 \\
\hline $0414+009$ & 0.287 & $\mathrm{H}$ & $16.08 \pm 0.05$ & $17.49 \pm 0.02$ & -24.77 & -23.71 & $4.70 \pm 0.50$ & 20.31 \\
\hline $0419+194$ & 0.512 & $\mathrm{H}$ & $19.53 \pm 0.17$ & $21.05 \pm 0.15$ & -22.80 & -22.22 & $0.40 \pm 0.07$ & 2.47 \\
\hline $0502+675$ & 0.314 & $\mathrm{H}$ & $17.33 \pm 0.10$ & $18.86 \pm 0.09$ & -23.75 & -22.62 & $0.60 \pm 0.07$ & 2.76 \\
\hline 0506-039 & 0.304 & $\mathrm{H}$ & $18.73 \pm 0.15$ & $18.35 \pm 0.01$ & -22.27 & -23.03 & $0.60 \pm 0.05$ & 7.19 \\
\hline $0521-365$ & 0.055 & $\mathrm{~L}$ & $15.28 \pm 0.10$ & $14.60 \pm 0.01$ & -21.68 & -22.41 & $2.80 \pm 0.07$ & 3.00 \\
\hline $0548-322$ & 0.069 & $\mathrm{H}$ & $16.93 \pm 0.10$ & $14.62 \pm 0.01$ & -20.54 & -22.92 & $7.05 \pm 0.15$ & 9.31 \\
\hline $0607+710$ & 0.267 & $\mathrm{H}$ & $18.23 \pm 0.10$ & $17.83 \pm 0.02$ & -22.44 & -23.17 & $2.40 \pm 0.12$ & 9.85 \\
\hline $0706+591$ & 0.125 & $\mathrm{H}$ & $17.53 \pm 0.07$ & $15.94 \pm 0.01$ & -21.31 & -23.04 & $3.05 \pm 0.07$ & 6.84 \\
\hline $0735+178$ & 0.424 & $\mathrm{~L}$ & $16.58 \pm 0.07$ & $>20.44$ & -25.26 & $>-22.06$ & - & 0.0 \\
\hline $0737+744$ & 0.315 & $\mathrm{H}$ & $17.88 \pm 0.15$ & $18.01 \pm 0.08$ & -23.20 & -23.48 & $2.10 \pm 0.45$ & 9.68 \\
\hline $0806+524$ & 0.138 & $\mathrm{H}$ & $15.98 \pm 0.02$ & $16.62 \pm 0.01$ & -23.09 & -22.61 & $1.45 \pm 0.03$ & 3.53 \\
\hline $0823+033$ & 0.506 & $\mathrm{~L}$ & $17.78 \pm 0.11$ & $>20.18$ & -24.52 & $>-23.04$ & - & 0.0 \\
\hline $0828+493$ & 0.548 & $\mathrm{~L}$ & $18.93 \pm 0.12$ & $20.26 \pm 0.10$ & -23.57 & -23.31 & $0.65 \pm 0.10$ & 4.16 \\
\hline $0829+046$ & 0.180 & $\mathrm{~L}$ & $15.88 \pm 0.07$ & $16.94 \pm 0.04$ & -23.83 & -22.98 & $4.30 \pm 0.75$ & 13.06 \\
\hline $0851+202$ & 0.306 & $\mathrm{~L}$ & $14.99 \pm 0.06$ & $>18.53$ & -26.02 & $>-22.88$ & - & 0.0 \\
\hline $0927+500$ & 0.188 & $\mathrm{H}$ & $17.48 \pm 0.30$ & $17.62 \pm 0.05$ & -22.33 & -22.40 & $2.00 \pm 0.45$ & 6.29 \\
\hline $0954+658$ & 0.367 & $\mathrm{~L}$ & $16.08 \pm 0.06$ & $>19.60$ & -25.39 & $>-22.40$ & - & 0.0 \\
\hline $0958+210$ & 0.344 & $\mathrm{H}$ & $21.48 \pm 0.40$ & $18.93 \pm 0.01$ & -19.82 & -22.84 & $0.82 \pm 0.04$ & 4.01 \\
\hline $1011+496$ & 0.2 & $\mathrm{H}$ & $15.88 \pm 0.05$ & $17.30 \pm 0.02$ & -24.08 & -22.89 & $1.80 \pm 0.12$ & 5.94 \\
\hline $1028+511$ & 0.361 & $\mathrm{H}$ & $16.48 \pm 0.10$ & $18.55 \pm 0.08$ & -24.95 & -23.40 & $1.80 \pm 0.35$ & 9.08 \\
\hline $1104+384$ & 0.031 & $\mathrm{H}$ & $13.78 \pm 0.08$ & $13.29 \pm 0.02$ & -21.90 & -22.41 & $3.95 \pm 0.05$ & 2.45 \\
\hline $1133+161$ & 0.460 & $\mathrm{H}$ & $20.28 \pm 0.18$ & $19.83 \pm 0.04$ & -21.77 & -22.99 & $1.55 \pm 0.23$ & 9.05 \\
\hline $1136+704$ & 0.045 & $\mathrm{H}$ & $16.15 \pm 0.04$ & $14.45 \pm 0.02$ & -20.35 & -22.10 & $3.10 \pm 0.02$ & 2.75 \\
\hline $1212+078$ & 0.136 & $\mathrm{H}$ & $16.38 \pm 0.10$ & $16.02 \pm 0.01$ & -22.66 & -23.17 & $3.40 \pm 0.10$ & 8.19 \\
\hline $1215+303$ & 0.130 & $\mathrm{H}$ & $14.55 \pm 0.01$ & $15.99 \pm 0.01$ & -24.38 & -23.08 & $8.35 \pm 0.20$ & 19.36 \\
\hline $1218+304$ & 0.182 & $\mathrm{H}$ & $15.68 \pm 0.10$ & $17.12 \pm 0.03$ & -24.05 & -22.82 & $2.60 \pm 0.30$ & 7.97 \\
\hline $1221+245$ & 0.218 & $\mathrm{H}$ & $16.89 \pm 0.05$ & $18.63 \pm 0.06$ & -23.28 & -21.79 & $1.25 \pm 0.25$ & 4.42 \\
\hline $1229+643$ & 0.164 & $\mathrm{H}$ & $18.03 \pm 0.30$ & $16.38 \pm 0.01$ & -21.45 & -23.29 & $2.00 \pm 0.07$ & 5.63 \\
\hline $1248-296$ & 0.370 & $\mathrm{H}$ & $18.83 \pm 0.08$ & $18.87 \pm 0.02$ & -22.66 & -23.15 & $1.10 \pm 0.05$ & 5.63 \\
\hline $1255+244$ & 0.141 & $\mathrm{H}$ & $17.08 \pm 0.05$ & $16.72 \pm 0.01$ & -22.05 & -22.57 & $2.50 \pm 0.05$ & 6.21 \\
\hline $1402+041$ & 0.340 & $\mathrm{H}$ & $16.38 \pm 0.01$ & $>19.38$ & -24.89 & $>-22.36$ & - & 0.0 \\
\hline $1407+595$ & 0.495 & $\mathrm{H}$ & $18.84 \pm 0.05$ & $19.04 \pm 0.05$ & -23.40 & -24.08 & $1.75 \pm 0.38$ & 10.63 \\
\hline $1418+546$ & 0.152 & $\mathrm{~L}$ & $15.68 \pm 0.06$ & $16.10 \pm 0.02$ & -23.62 & -23.37 & $3.65 \pm 0.11$ & 9.65 \\
\hline $1426+428$ & 0.129 & $\mathrm{H}$ & $17.38 \pm 0.20$ & $16.14 \pm 0.01$ & -21.53 & -22.92 & $2.25 \pm 0.08$ & 5.18 \\
\hline $1440+122$ & 0.162 & $\mathrm{H}$ & $16.93 \pm 0.12$ & $16.71 \pm 0.02$ & -22.52 & -22.93 & $3.90 \pm 0.25$ & 10.87 \\
\hline $1458+224$ & 0.235 & $\mathrm{H}$ & $15.78 \pm 0.08$ & $17.80 \pm 0.05$ & -24.57 & -22.83 & $3.20 \pm 0.80$ & 11.96 \\
\hline $1514-241$ & 0.049 & $\mathrm{H}$ & $14.48 \pm 0.12$ & $14.45 \pm 0.01$ & -22.22 & -22.30 & $3.70 \pm 0.10$ & 3.55 \\
\hline
\end{tabular}


Table 4 -Continued

\begin{tabular}{cccccccccc}
\hline \hline Name & $\mathrm{z}$ & Type $^{\mathrm{a}}$ & $\mathrm{m}_{R}$ nucleus $^{\mathrm{b}}$ & $\mathrm{m}_{R}$ host $^{\mathrm{c}}$ & $\mathrm{m}_{R}$ nucl. $^{\mathrm{d}}$ & $\mathrm{m}_{R}$ host $^{\mathrm{e}}$ & $\mathrm{r}_{e}(\operatorname{arcsec})^{\mathrm{f}}$ & $\mathrm{r}_{e}(\mathrm{kpc})^{\mathrm{g}}$ \\
\hline $1534+014$ & 0.312 & $\mathrm{H}$ & $19.08 \pm 0.15$ & $18.16 \pm 0.02$ & -21.98 & -23.31 & $2.00 \pm 0.10$ & 9.16 \\
$1704+604$ & 0.280 & $\mathrm{H}$ & $21.08 \pm 0.35$ & $18.69 \pm 0.01$ & -19.71 & -22.45 & $0.85 \pm 0.03$ & 3.61 \\
$1728+502$ & 0.055 & $\mathrm{H}$ & $16.43 \pm 0.10$ & $15.49 \pm 0.02$ & -20.53 & -21.52 & $3.15 \pm 0.05$ & 3.37 \\
$1749+096$ & 0.320 & $\mathrm{~L}$ & $16.88 \pm 0.05$ & $18.82 \pm 0.10$ & -24.24 & -22.73 & $3.00 \pm 0.80$ & 13.97 \\
$1757+703$ & 0.407 & $\mathrm{H}$ & $18.43 \pm 0.14$ & $19.58 \pm 0.25$ & -23.31 & -22.77 & $0.85 \pm 0.50$ & 4.62 \\
$1807+698$ & 0.051 & $\mathrm{~L}$ & $14.95 \pm 0.25$ & $13.87 \pm 0.02$ & -21.84 & -22.97 & $2.10 \pm 0.10$ & 2.09 \\
$1853+671$ & 0.212 & $\mathrm{H}$ & $19.48 \pm 0.10$ & $18.19 \pm 0.01$ & -20.63 & -22.17 & $1.50 \pm 0.08$ & 5.18 \\
$1959+650$ & 0.048 & $\mathrm{H}$ & $15.38 \pm 0.10$ & $14.92 \pm 0.02$ & -21.27 & -21.78 & $5.10 \pm 0.10$ & 4.80 \\
$2005-489$ & 0.071 & $\mathrm{H}$ & $12.73 \pm 0.01$ & $14.52 \pm 0.01$ & -24.81 & -23.09 & $5.65 \pm 0.08$ & 7.65 \\
$2007+777$ & 0.342 & $\mathrm{~L}$ & $18.03 \pm 0.10$ & $19.03 \pm 0.10$ & -23.26 & -22.73 & $3.30 \pm 0.90$ & 16.07 \\
$2143+070$ & 0.237 & $\mathrm{H}$ & $18.21 \pm 0.11$ & $17.89 \pm 0.02$ & -22.16 & -22.76 & $2.10 \pm 0.15$ & 7.90 \\
$2200+420$ & 0.069 & $\mathrm{~L}$ & $13.58 \pm 0.05$ & $15.37 \pm 0.02$ & -23.89 & -22.17 & $4.80 \pm 0.40$ & 6.33 \\
$2201+044$ & 0.027 & $\mathrm{~L}$ & $17.18 \pm 0.05$ & $13.74 \pm 0.01$ & -18.18 & -21.64 & $6.78 \pm 0.08$ & 3.68 \\
$2254+074$ & 0.190 & $\mathrm{~L}$ & $16.94 \pm 0.12$ & $16.61 \pm 0.02$ & -22.90 & -23.44 & $4.90 \pm 0.35$ & 15.54 \\
$2326+174$ & 0.213 & $\mathrm{H}$ & $17.63 \pm 0.11$ & $17.56 \pm 0.03$ & -22.49 & -22.81 & $1.80 \pm 0.15$ & 6.24 \\
$2344+514$ & 0.044 & $\mathrm{H}$ & $16.83 \pm 0.05$ & $14.01 \pm 0.01$ & -19.62 & -22.49 & $5.93 \pm 0.02$ & 5.14 \\
$2356-309$ & 0.165 & $\mathrm{H}$ & $17.28 \pm 0.13$ & $17.21 \pm 0.02$ & -22.21 & -22.47 & $1.85 \pm 0.10$ & 5.23 \\
\hline
\end{tabular}

aSED type: $\mathrm{H}=\mathrm{HBL}$ with $F_{1 k e V} / F_{5 G H z}>5.5$, and $\mathrm{L}=\mathrm{LBL}$, with $F_{1 k e V} / F_{5 G H z}<5.5$.

${ }^{\mathrm{b}}$ Apparent R-band magnitude of nucleus from best-fit PSF + de Vaucouleurs model.

${ }^{\mathrm{c}}$ Apparent R-band magnitude of host galaxy from best-fit PSF + de Vaucouleurs model.

dAbsolute R-band magnitude of nucleus, K corrected assuming $\alpha=1$.

e Absolute R-band magnitude of host galaxy, K corrected assuming early-type spectrum.

${ }^{\mathrm{f}} \mathrm{De}$ Vaucouleurs effective radius in arcseconds.

g De Vaucouleurs effective radius in kiloparsecs, assuming $q_{0}=0$.

\begin{tabular}{lcc}
$\begin{array}{l}\text { Assumed } \\
z_{\text {form }}\end{array}$ & $\begin{array}{c}\text { Slope } \\
(\mathrm{K} \text {-band mag/z) }\end{array}$ & $\begin{array}{c}\text { Intercept } \\
\text { (K-band mag) }\end{array}$ \\
\hline 2.8 & $-1.37 \pm 0.30$ & $-24.97 \pm 0.11$ \\
1.8 & $-0.98 \pm 0.29$ & $-25.02 \pm 0.10$ \\
1.3 & $-0.65 \pm 0.28$ & $-25.04 \pm 0.10$ \\
\hline
\end{tabular}

Table 5: Regression analysis results for absolute luminosity evolution based on the range of evolution model fits to the Hubble diagram. 
Fig. 1.- Central regions of STIS F28×50LP images right, with contour plots left.

Fig. 2.- Two-dimensional and three-dimensional images of the composite stellar PSF constructed from archival STIS F28×50LP images of stars.

Fig. 3.- Azimuthally averaged profiles of STIS images. The upper left and right panels show the best-fit de Vaucouleur + PSF models and exponential disk + PSF models respectively. Middle panels show zoomed-in profiles, inset with $1,2 \& 3 \sigma \chi^{2}$ contours projected on $m_{\text {host }}-r_{e}$ plane (for cases where the host galaxy was confidently detected.) Lower left panels show the best-fit dual-component models and lower right panels show the best-fit PSF-only models. For 1308+326, only the best PSF + exponential disk upper and lower left and PSF-only right profiles are shown, while for 2240-260 the best PSF-only profile is shown.

Fig. 4.- BL Lac images with best-fit models subtracted - top: unsubtracted image; middle: PSF subracted, normalized according to best-fit de Vaucouleurs model; bottom: best PSF + de Vaucouleurs model subtracted. For 1308+326 and 2240-260, the unsubtracted image (top) and best-fit PSF subtraction assuming no host galaxy (bottom) are shown. The arrow next to the object name indicates north and east. In the case of $0235+164$, we chose a gray scale to bring out the detail in the surrounding structure, and so the marginal host galaxy is difficult to make out.

Fig. 5.- Hubble diagram for BL Lac host galaxies, in F28×50LP magnitudes, corrected for galactic extinction. The large points are the high-z sample: STIS targets from this study. The small points are the low-z sample: WFPC2 snapshot targets with $z<0.6$ from Urry et al. (2000). Also shown are the expected tracks of a non-evolving stellar population with an early-type spectrum $(B-V=0.96$; dashed line $)$, and a late-type spectrum $(B-V=0.57$; dotted line).

Fig. 6.- Hubble diagrams of high-z (large points) and low-z (small points) BL Lac host galaxies. Top shows the best-fit passively evolving model, with $z_{\text {form }}=1.8$ (solid line), and the $z_{\text {form }}=5$ passive model (dashed line). Middle shows the best-fit non-evolving model which also fits the low-z colour constraint of $R-H>2$, with age $=5$ Gyrs (solid line), as well as the age $=1$ Gyrs (dashed line) and age $=0.02$ Gyrs (dotted line) non-evolving models. This latter extremely young population is needed for a non-evolving population to fit the data. Bottom shows a dual population (solid line), in which $98 \%$ of the mass is evolving passively from $z_{\text {form }}=5$, and $2 \%$ is undergoing active star formation with a constant age of 1 Gyr down to $z=0.6$, after which it evolves passively. 
Fig. 7.- Hubble diagrams of high-z (large points) and low-z (small points) BL Lac host galaxies with evolutionary models including dust and metallicity evolution. Top left shows a 0.8 Gyr non-evolving population with dust increasing linearly to an extreme optical depth of $\tau_{V}(0)=8$ between $z=0.8$ to 0.4 (solid line), giving a reasonable fit to the data. The dashed line shows dust increasing to $\tau_{V}(0)=2$, and the dotted line shows the model with no dust, both yielding poor fits. Top right shows a $z_{\text {form }}=5$ passive population with dust increasing from $\tau_{V}(0)=0$ to 10 by low redshift, producing a reasonable fit, while an increase to $\tau_{V}(0)=2$ (dashed line) or a model with no dust (dotted line) produce poor fits. Bottom left shows the non-evolving population with metallicity increasing from 0.02 solar to 1 solar at $z=0.6$. The solid line produces a reasonable fit with dust also increasing to $\tau_{V}(0)=5$ by low redshifts. An increase to $\tau_{V}(0)=2$ (dashed line) produces a marginal fit. The dotted line shows the model with no change in dust or metallicity. Bottom right is the same as bottom left, but with a $z_{\text {form }}=5$ passively evolving population, which requires the same dust and metallicity evolution to fit the data.

Fig. 8. - Absolute K-band magnitude versus redshift for high-z (large dots) and low-z (small dots) BL Lac host galaxies. These are K corrected assuming a redshift-dependent spectrum, determined using the evolution model which provided the best fit to the Hubble diagram (passive evolution with $z_{\text {form }}=1.8$; see $§ 4.3$ ). Also plotted are the best-fit regression lines, derived using survival analysis to account for the upper limits. The solid line shows the best fit for $z_{\text {form }}=1.8 \mathrm{~K}$ corrections, while the other lines represent the $3 \sigma$ upper and lower limits to the slope, determined assuming $z_{\text {form }}=2.8 \mathrm{~K}$ corrections (dashed line) and $z_{\text {form }}=1.3$ $\mathrm{K}$ corrections (dotted line). 
This figure "f1a.jpg" is available in "jpg" format from: http://arxiv.org/ps/astro-ph/0411099v1 
This figure "f1b.jpg" is available in "jpg" format from: http://arxiv.org/ps/astro-ph/0411099v1 
This figure "f1d.jpg" is available in "jpg" format from: http://arxiv.org/ps/astro-ph/0411099v1 
This figure "f1e.jpg" is available in "jpg" format from: http://arxiv.org/ps/astro-ph/0411099v1 
This figure "f1f.jpg" is available in "jpg" format from: http://arxiv.org/ps/astro-ph/0411099v1 
This figure "f1g.jpg" is available in "jpg" format from: http://arxiv.org/ps/astro-ph/0411099v1 
This figure "f1h.jpg" is available in "jpg" format from: http://arxiv.org/ps/astro-ph/0411099v1 
This figure "fli.jpg" is available in "jpg" format from: http://arxiv.org/ps/astro-ph/0411099v1 
This figure "f2.jpg" is available in "jpg" format from: http://arxiv.org/ps/astro-ph/0411099v1 
This figure "f3a.gif" is available in "gif" format from: http://arxiv.org/ps/astro-ph/0411099v1 
This figure "f3b.gif" is available in "gif" format from: http://arxiv.org/ps/astro-ph/0411099v1 
This figure "f3c.gif" is available in "gif" format from: http://arxiv.org/ps/astro-ph/0411099v1 
This figure "f3d.gif" is available in "gif" format from: http://arxiv.org/ps/astro-ph/0411099v1 
This figure "f3e.gif" is available in "gif" format from: http://arxiv.org/ps/astro-ph/0411099v1 
This figure "f3f.gif" is available in "gif" format from: http://arxiv.org/ps/astro-ph/0411099v1 
This figure "f3g.gif" is available in "gif" format from: http://arxiv.org/ps/astro-ph/0411099v1 
This figure "f3h.gif" is available in "gif" format from: http://arxiv.org/ps/astro-ph/0411099v1 
This figure "f3i.gif" is available in "gif" format from: http://arxiv.org/ps/astro-ph/0411099v1 
This figure "f4a.jpg" is available in "jpg" format from: http://arxiv.org/ps/astro-ph/0411099v1 
This figure "f4b.jpg" is available in "jpg" format from: http://arxiv.org/ps/astro-ph/0411099v1 
This figure "f4c.jpg" is available in "jpg" format from: http://arxiv.org/ps/astro-ph/0411099v1 
This figure "f4d.jpg" is available in "jpg" format from: http://arxiv.org/ps/astro-ph/0411099v1 
This figure "f4e.jpg" is available in "jpg" format from: http://arxiv.org/ps/astro-ph/0411099v1 
This figure "f4f.jpg" is available in "jpg" format from: http://arxiv.org/ps/astro-ph/0411099v1 
This figure "f4g.jpg" is available in "jpg" format from: http://arxiv.org/ps/astro-ph/0411099v1 
This figure "f4h.jpg" is available in "jpg" format from: http://arxiv.org/ps/astro-ph/0411099v1 
This figure "f4i.jpg" is available in "jpg" format from: http://arxiv.org/ps/astro-ph/0411099v1 
This figure "f5.gif" is available in "gif" format from: http://arxiv.org/ps/astro-ph/0411099v1 
This figure "f6.gif" is available in "gif" format from: http://arxiv.org/ps/astro-ph/0411099v1 
This figure "f7.gif" is available in "gif" format from: http://arxiv.org/ps/astro-ph/0411099v1 
This figure "f8.gif" is available in "gif" format from: http://arxiv.org/ps/astro-ph/0411099v1 